\title{
Continuum radiation from active galactic nuclei
}

\author{
Joel N. Bregman \\ Astronomy Department, Dennison Building, University of Michigan, \\ Ann Arbor, MI 48109-1090, USA
}

Received May 21, 1990

Summary. Active galactic nuclei (AGN) can be divided into two broad classes, where the emitted continuum power is dominated either by thermal emission (radio-quiet AGN), or by nonthermal emission (blazars). Emission in the 0.01$1 \mu \mathrm{m}$ range is the primary contributor to the bolometric luminosity and is probably produced through thermal emission from an accretion disk, modified by electron scattering and general relativistic effects. The $1-1000 \mu \mathrm{m}$ continuum, the second most important contributor to the power, is generally dominated by thermal emission from dust with a range of temperatures from $\sim 40 \mathrm{~K}$ to $\sim 1000-2000 \mathrm{~K}$. The dust is probably reemitting $0.01-0.3 \mu \mathrm{m}$ continuum emission, previously absorbed in an obscuring cone (or torus) or an extended disk. The $1-10 \mathrm{keV}$ X-ray emission is rapidly variable and originates in a small region. This emission may be produced through Compton scattering by hot thermal electrons surrounding an accretion disk, although the observations are far from being definitive. The weak radio emission, which is due to the nonthermal synchrotron process, is usually elongated in the shape of jets and lobes (a core may be present too), and is morphologically distinct from the radio emission of starburst galaxies.

In the blazar class, the radio through ultraviolet emission is decidedly nonthermal, and apparently is produced through the synchrotron process in an inhomogeneous plasma. The plasma probably is moving outward at relativistic velocities within a jet in which the Lorentz factor of bulk motion (typically 2-6) increases outward. This is inferred from observations indicating that the opening angle becomes progressively larger from the radio to the optical to the X-ray emitting regions. Shocks propagating along the jet may be responsible for much of the flux variability. In sources where the X-ray continuum is not a continuation of the optical-ultraviolet synchrotron emission, some objects show variability consistent with Compton scattering by relativistic electron in a large region (in BL Lacertae), while other objects produce their X-ray emission in a compact region, possibly suggesting pair production.

When orientation effects are included, all AGN may be decomposed into a radio-quiet AGN, a blazar, or a combination of the two. Radio-quiet AGN appear to have an obscuring cone or torus containing the broad emission line 
clouds and an ionizing source. Most likely, the (non-relativistic) directional effects of this obscuring region give rise to the difference between Seyfert 1 and 2 galaxies or narrow and broad line radio galaxies. For different orientations of the nonthermal jet, relativistic Doppler boosting can produce BL Lacertae objects or FR I radio galaxies, or at higher jet luminosities, flat-spectrum highpolarization quasars or FR II radio galaxies.

Key words: BL Lacertae objects - galaxies: nuclei of - galaxies: Seyfert - galaxies: radio - quasars: general

\section{Introduction}

One of the most challenging problems in the area of active galactic nuclei has been the development of a deep understanding of continuum emission. In the broadest sense, the important issues are the mechanisms responsible for the radiation, the kinematics and spatial distribution of the emitting regions, and the connection of the continuum emission to the central engine and host galaxy. Progress in answering these issues has been slow, partly because the continuum emission extends over an enormously broad range of frequencies (at least $10^{6}-10^{23} \mathrm{~Hz}$ ), of which many wavebands were unaccessible until this past decade. Furthermore, the broad spectral signatures that one normally searches for are not present (e.g., a single temperature black-body spectrum of a star), causing investigators to try to identify the critical characteristics that could be compared to newly developed theories.

Fortunately, the improvement in the sensitivity of instruments in a variety of wavebands (millimeter, submillimeter, near infrared), and the opening of other wavebands through space observatories (far infrared, X-ray) have provided the more complete observational picture that has been essential in leading to the progress in answering fundamental questions.

Perhaps the greatest change that has occurred in this field has been the evidence showing that there are two general types of $A G N$, those in which most of the emitted power is thermal, and those in which it is nonthermal. This perception is vividly contrasted with the view a decade ago in which there was broad agreement that nonthermal processes led to the continuum emission from nearly all AGN. This new order is an important step in the effort to examine objects according to physical rather than morphological type. Consequently, the first half of this review is devoted to the objects in which the emission is largely thermal, typically the radio-quiet $A G N$, while the second half of the review discusses the objects dominated by nonthermal emission, the blazar class. We discuss the evidence separating these two classes of objects, and the most likely underlying physical mechanisms. In each of the separate sections and subsections, we try to summarize the state of affairs, identify the central issues, and occasionally, suggest observational tests and theoretical calculations to be carried out.

\section{Thermally emitting active galactic nuclei}

A decade ago, there was nearly universal agreement that the continuum emission from quasars, Seyfert 1 galaxies, and broad-line radio galaxies was entirely of 
nonthermal origin. The weight of evidence has shifted so dramatically that it can be effectively argued that nearly all of the power, and all the emission in the $0.01-1000 \mu \mathrm{m}$ region is emitted by thermal processes. We review the basic properties of the continuum emission, why thermal emission is a favored explanation, and whether orientation and evolutionary effects are critical to the appearance of an $\mathrm{AGN}$.

There are a host of different categories of active galactic nuclei, although for some purposes, they can be sensibly grouped together (e.g., Lawrence 1987). In some cases, the differences between classes of objects is probably caused by the ease with which the underlying galaxy is detected. For example, Seyfert 1 galaxies are probably low luminosity counterparts of radio quiet quasars. For the purposes of this review, we will treat the low and high luminosity radio quiet AGN (Seyfert 1s and quasars) as a single type of object. We will also discuss the evidence that other classes of objects, such as Seyfert $2 \mathrm{~s}$, are radio quiet $\mathrm{AGN}$ seen from a special orientation. The radio-loud $\mathrm{AGN}$ that are not blazars share many important continuum properties of radio-quiet AGN. However, they are especially complex objects because they show evidence for a mixture of both types of continuum emission properties. Their role in the overall picture will be considered last, once the more basic properties are identified. We begin this part of the review by discussing in detail the continuous spectrum of a typical $\mathrm{AGN}$, and then proceed to examine proposed models for the most prominent features.

\subsection{The spectrum of a typical $A G N$}

For a typical radio-quiet AGN, a plot of the power per logarithmic bandwidth $\left(\log v F_{v}\right.$ vs $\left.\log v\right)$ reveals that most of the power emerges in the range $12<\log v$ $<18$, where $v$ has units of $\mathrm{Hz}$ (Fig. 1). Our discussion of the typical spectrum will progress with increasing frequency, beginning with the radio region. Even radio-quiet AGN have radio luminosities considerably greater than that of an ordinary spiral galaxy, although due to their great distances, their flux densities may be difficult to measure (Seyferts are discussed by Wilson and Meurs 1982; Ulvestad and Wilson 1984; Meurs and Wilson 1984; a complete sample of quasars was analyzed by Kellermann et al. 1989). The radio emission in most objects have "steep" spectra (e.g., Wilsson and Heckman 1985), with typical slopes of $-1.1<\alpha<-0.4$, although a few objects have compact, flat spectrum cores (throughout this review, $\alpha$ is defined by $F_{v} \propto v^{\alpha}$ ). A typical steep radio spectrum does not connect smoothly into the submillimeter-infrared region, suggesting that it is a distinct component. Relative to the bolometric luminosity of an object, the power emitted in the radio region is small, even for the radio loud sources.

At infrared wavelengths, the spectrum rises extremely sharply in the 100 $1000 \mu \mathrm{m}$ range, peaking between $10 \mu \mathrm{m}$ and $100 \mu \mathrm{m}$ (in $v F_{v}$; Miley et al. 1985; Edelson and Malkan 1986; Carleton et al. 1987; Chini et al. 1987; Antonucci and Barvainis 1988; Engargiola et al. 1988; Barvainis and Antonucci 1989; Chini et al. 1989; Krugel et al. 1989; Sanders et al. 1989). This infrared part of the spectrum, which is generally convex, typically contains a third of the bolometric luminosity of the source. However, a broad range of behavior exists, with some objects emitting from $10-90 \%$ of their luminosity in the infrared region. 


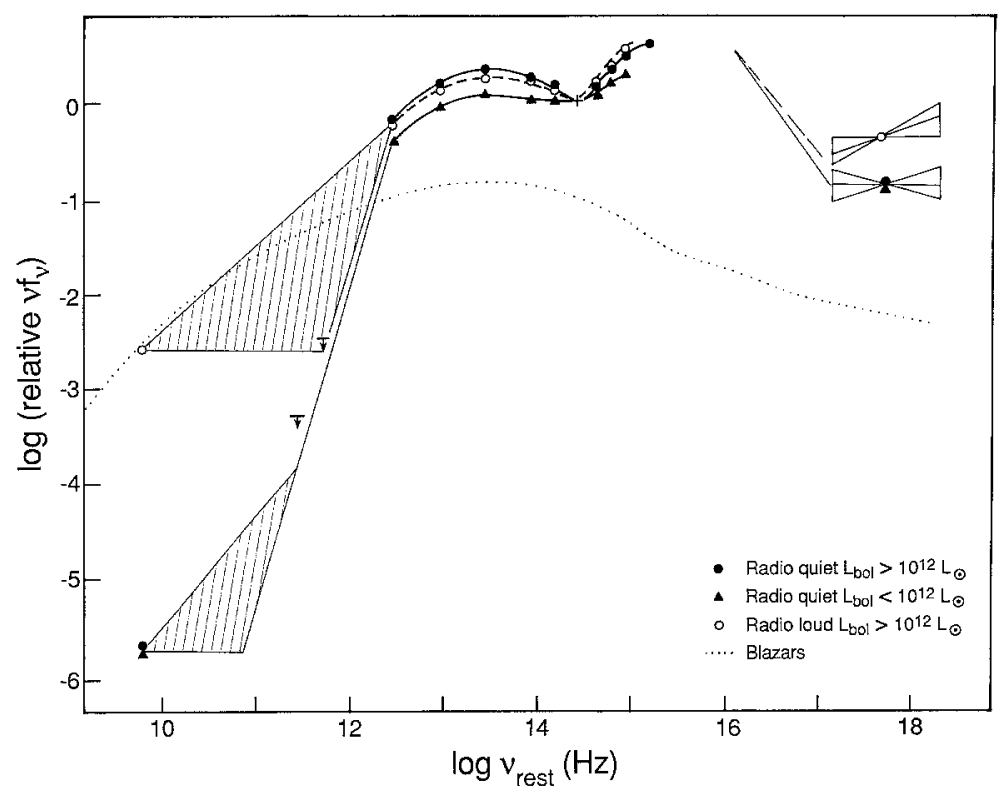

Fig. 1. The mean continua of blazars and two types of quasars from the radio through X-ray regions (from Sanders et al. 1989). The continuum of radio quiet quasars suggest that four separate emission components are present: the radio $\left(10^{9}-10^{11.5} \mathrm{~Hz}\right)$, the submillimeter infrared $\left(10^{12}-10^{14.5} \mathrm{~Hz}\right)$, the near infrared to soft X-ray $\left(10^{14.5}-10^{17} \mathrm{~Hz}\right)$, and the X-ray $\left(>10^{17} \mathrm{~Hz}\right)$ regions. Such distinct features are not present in blazar continua

The limited spectral resolution has prevented the identification of narrow continuum features in the $12-100 \mu \mathrm{m}$ range, but narrow band near infrared photometry has been able to distinguish a bump in the 3-5 $\mu \mathrm{m}$ range (Edelson and Malkan 1986). Despite the limitations associated with the study of near infrared features in intrinsically weak objects, Edelson and Malkan (1986) obtained observations in the $1-10 \mu \mathrm{m}$ region for a heterogeneous sample of 29 AGN (IRAS data was also included in the study). They characterized the spectra by a number of simple components, such as a power-law in the near infrared region. The fit to the data was improved considerably with the addition of a $5.2 \mu \mathrm{m}$ bump, a feature which was found to lie at nearly the same rest frequency with remarkable consistency (a peak frequency of $58 \pm 11 \mathrm{THz}$ and a width of 3.3 octaves). The central frequency of this near infrared bump does depend upon its assumed shape and the form of the underlying continuum. Carleton et al. (1987) show that in 3C 273, the peak frequency may fall at $3.5 \mu \mathrm{m}$. Despite this uncertainty, a near infrared bump appears to be present and may provide an important clue in completing the picture of continuum emission.

Many of the broad-band spectra show a local minimum near $1 \mu \mathrm{m}$ (rest frame; Figs. 1 and 2), a feature which is of key importance in the debate surrounding the origin of the emission. This minimum is particularly evident in the high luminosity radio-quiet quasars, such as those in the PG sample (Sanders et al. 1989) or in Seyfert galaxies (McAlary and Rieke 1988). It is also present in radio loud AGN, but is less prominent in the lower luminosity radio-quiet AGN. The weakening of this feature in low luminosity objects in the study 


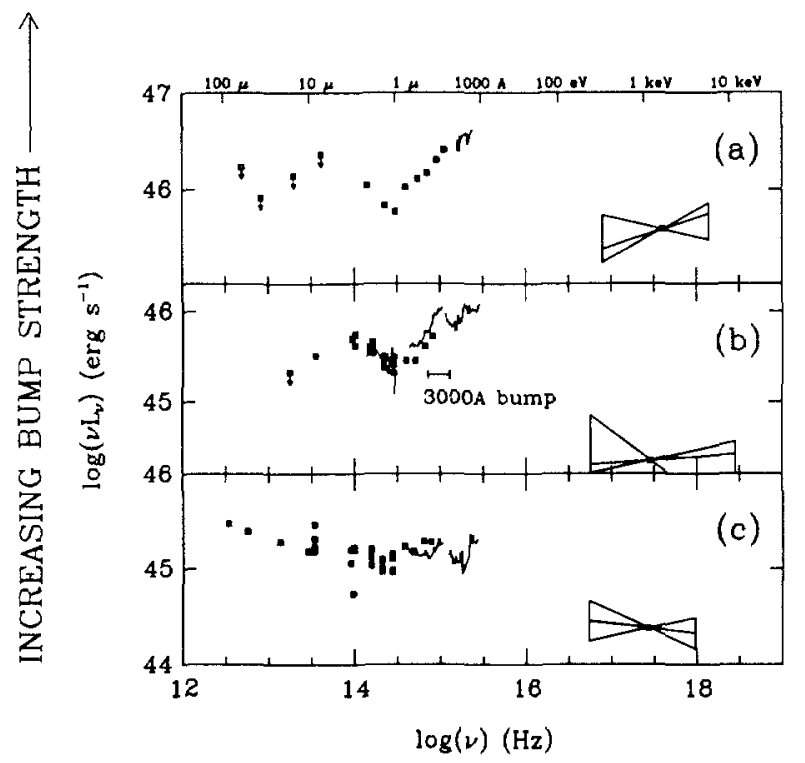

Fig. 2. The $100 \mu \mathrm{m}$ to $10 \mathrm{keV}$ continua of three quasars (from McDowell et al. 1989). The rising continuum in the $1 \mu \mathrm{m}$ to $1000 \mathrm{~A}$ region contains most of the observed power in $3 \mathrm{C} 263$ and PG $1116+215$ and suggests that the peak power occurs in the extreme ultraviolet region. The relative power contained in this big blue bump can vary widely, as seen in PG $1613+658$

of Sanders et al. (1989) is probably due to contamination by the underlying galaxy, which emit much of their power near $1.5 \mu \mathrm{m}$.

The power per logarithmic bandwidth rises continuously from $1 \mu \mathrm{m}$ into the optical and infrared region for most objects. Several components contribute to this rise, some of which are associated with the broad lines, such as Balmer continuum emission and Fe II emission (see, e.g., review by Osterbrock and Mathews 1986). The contribution from the Balmer continuum occurs in the range 2700-3800 $\AA$, while the forest of Fe II emission lines creates an irregularly shaped pseudo-continuum that is prominent at $1800-3500 \AA$ (Wills et al. 1985 ; Fig. 3). These two features, collectively referred to as the " $3000 \AA$ bump", sit upon a stronger continuum, which continues to rise into the vacuum ultraviolet (in $v F_{v}$ vs $v$; Fig. 2) and is sometimes referred to as the ("big") blue bump. The ultraviolet end of the blue bump is defined by data obtained with the International Ultraviolet Explorer, which is insensitive to radiation below about $1200 \AA$. A flattening or a turn-down is sometimes seen in the shortest accessible ultraviolet part of dereddened spectra, suggesting that the peak in the power can occur in the 1200-2000 $\AA$ range (Malkan 1988; Sun and Malkan 1989). However, in many objects, the power continues to rise into and peak in the extreme ultraviolet region $(1200 \AA-100 \AA$, or $10 \mathrm{eV}-100 \mathrm{eV})$, providing the dominant contribution to the bolometric luminosity (Elvis et al. 1990; Fig. 2).

Quasar continuum emission can be observed again for energies above $\approx 0.3 \mathrm{keV}$, which is where the Galaxy becomes transparent once more. At these energies, the useful detectors are generally of two types, a "soft" X-ray detector, sensitive to photons in the $0.1 \mathrm{keV}$ to a few $\mathrm{keV}$ range, and a "harder" $\mathrm{X}$-ray detector, which typically operates between $2 \mathrm{keV}$ and $6-30 \mathrm{keV}$. The Imaging Proportional Counter on the Einstein Observatory $(0.1-4 \mathrm{keV})$ had a spectral 


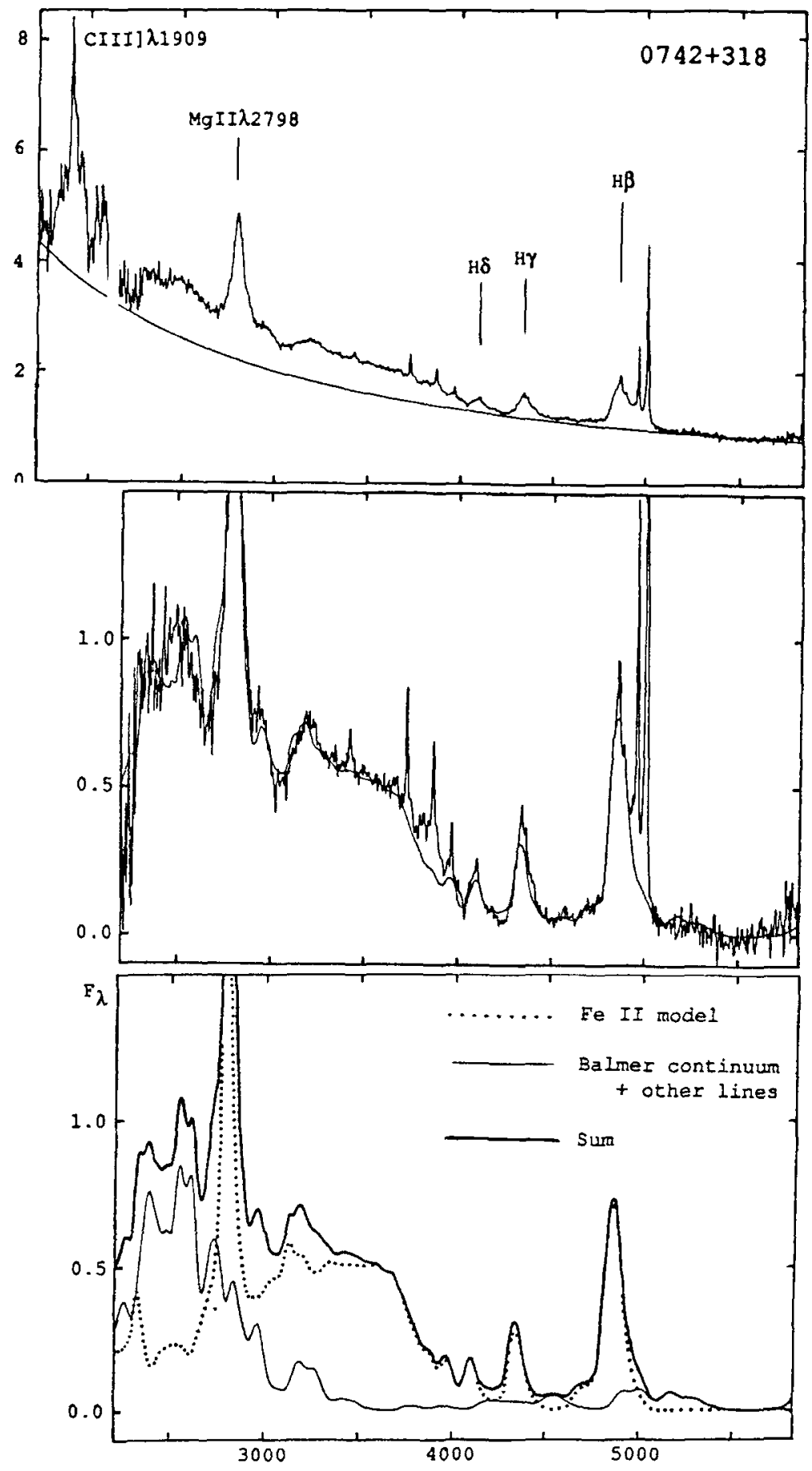

Fig. 3. The optical and ultraviolet spectrum of the quasar $0742+318$ (from Wills, Netzer, and Wills 1985). Balmer continuum emission and a forest of blended Fe II lines creates a pseudocontinuum that lies upon a rising underlying continuum (modeled here as a power-law) 


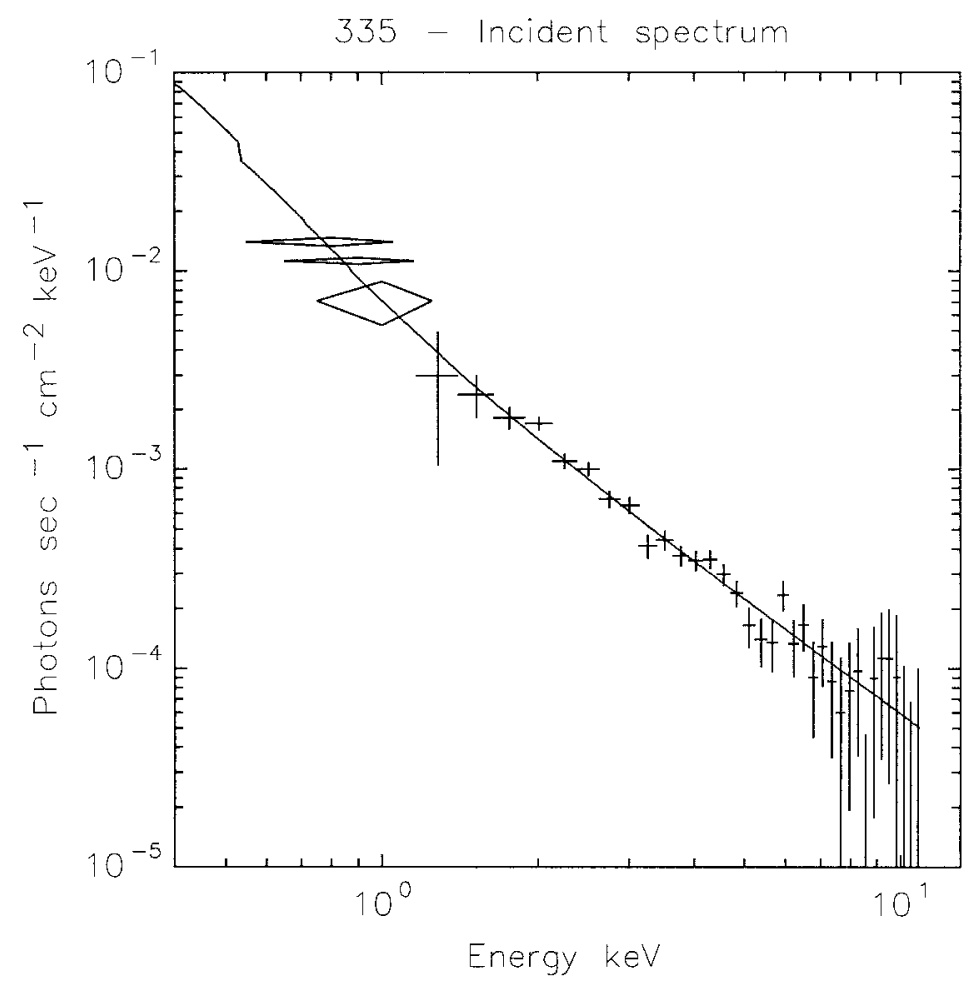

Fig. 4. The $0.5-10 \mathrm{keV}$ spectrum of the radio quiet object Mrk 335 (from Turner and Pounds 1988). The open diamonds, data taken wirth the LE on EXOSAT, show the rise at the lowest energy region which defines the soft X-ray excess. This soft excess may be the high frequency extension of the blue bump that peaks in the ultraviolet and extreme ultraviolet region

resolution of $\Delta E / E \approx 1$, so a spectrum could be divided into only a few independent bins. In the lowest energy range $(0.1-0.6 \mathrm{keV})$, extinction by galactic disk gas and by gas intrinsic to the $\mathrm{AGN}$ is an important effect. Upon correcting for absorption, a soft X-ray excess is found in several sources (Arnaud et al. 1985; Singh et al. 1985; Pounds et al. 1986; Wilkes and Elvis 1987). For example, in $3 \mathrm{C} \mathrm{263}$, the quantity $v F_{v}$ rises from the $1 \mathrm{keV}$ region to the lower energy cutoff near $0.1 \mathrm{keV}$ (McDowell et al. 1989). This effect has been studied in objects such as PG 1426+015 and PG 1211+143 (Elvis et al. 1985; Elvis et al. 1990, and references therein), where the power per logarithmic bandwidth rises into the unobservable extreme ultraviolet band from both the ultraviolet and soft $\mathrm{X}$-ray regions. The same effect is seen with slightly better definition in some objects observed with EXOSAT, which had a soft X-ray instrument that used broadband X-ray filters to provide modest spectral information. Two of the best examples of objects with soft X-ray excesses are Mrk 335 (Fig. 4), and NGC 5548 (Turner and Pounds 1988, 1989; review by Pounds 1989). The agreement in the results found with two different instruments is reaffirming and demonstrates that for some objects, most of the power emerges in the unobserved extreme ultraviolet region.

At energies exceeding $\approx 1 \mathrm{keV}$, or at all $\mathrm{X}$-ray energies when a soft excess is absent, the X-ray continuum can be represented by a power-law (review and 
discussion of spectral decompositions by Mushotzky 1987). In the $2-120 \mathrm{keV}$ band, the flux densities from AGN are low, so that only the X-ray bright objects have been studied. Several instruments operating in this spectral range were on HEAO-1, a scanning X-ray satellite telescope that surveyed the sky to a limiting flux density. This experiment produced samples that were well-defined by their X-ray properties, but inhomogeneous in their radio-optical properties. The $2-20 \mathrm{keV}$ continuum, corrected for absorption, is fit by a power law of slope $\alpha=-0.7$, with surprisingly little dispersion (26 objects; Mushotzky 1982, 1984). In the subset of 12 objects detectable at energies extending to $120 \mathrm{keV}$, the spectrum also had a slope of about -0.7 (Rothschild et al. 1983). The spectral slope in the $0.5-4.5 \mathrm{keV}$ range is not nearly so simple described. Using the hard X-ray selected sample that had define the "universal" X-ray slope of -0.7 , Turner and Pounds (1989) found the softer X-ray region $(2-10 \mathrm{keV})$ was of similar slope. However, for other samples of objects, a wider range of X-ray slopes is found (Halpern 1982; Petre et al. 1984; Reichert et al. 1985; Elvis et al. 1986; Pounds 1985, 1989; Kruper et al. 1990). In particular, from samples of objects observed with the Einstein Observatory, radio quiet AGN have an average soft X-ray slope near -1.0 , while the radio-loud objects have a mean slope of -0.5 (Elvis et al. 1986; Maccacaro et al. 1988; Sanders et al. 1989; Worrall 1989; Brunner et al. 1989); there is a larger dispersion about the mean value than for the hard X-ray selected sample. Relative to their emission at $1 \mu \mathrm{m}$, the radio-loud objects are more $\mathrm{X}$-ray luminous.

\subsection{The origin of the $X$-ray emission}

The origin of the X-ray emission is an important, but still poorly understood aspect of the continuum emission. The X-ray variability measurements, along with the rather uniform continuum shape above $2 \mathrm{keV}$ provide important clues that limit the possible models. Extensive time variability studies of the X-ray emission from Seyfert galaxies were caried out by EXOSAT "long looks", in which objects were monitored continuously for up to three days (reviewed by Pounds 1985; Pounds and McHardy 1988). Variations were seen on the shortest accessible timescales $(\sim 100 \mathrm{~s})$ for Mrk 335, NGC 4051, NGC 5506, NGC 5548, MCG 6-30-15, and MCG 8-11-11 (Lawrence et al. 1987; McHardy and Czerny 1987; Pounds and McHardy 1988; Matsuoka et al. 1989; Nandra et al. 1989; Done et al. 1990; Kaastra and Barr 1990), thereby demonstrating that the X-rays are produced in a small region. A related issue is whether the X-ray emission is thermal or nonthermal in origin. Although the observations are not definitive in this matter, the X-ray continuum, which in the range $3-100 \mathrm{keV}$ is well described by a single power-law $(\alpha=-0.7)$, is consistent with a nonthermal mechanism.

Within the picture where an accretion disk and supermassive black hole lie at the center of an AGN, the central region of this system is a natural site for the production of X-rays. Compton scattering toward the top of an accretion disk atmosphere, which derives its opacity from electrons, is known to give a hard X-ray tail (e.g., Shakura and Sunyaev 1973; Czerny and Elvis 1987; Laor et al. 1990). Maraschi and Molendi (1990) discuss a model in which the effective optical thickness falls below unity in the inner part of the accretion disk. For near-critical accretion rates and high viscosity, Comptonization of 
the free-free flux in this inner region produces a hard X-ray tail extending to $\sim 30 \mathrm{keV}$. In this, and other models where the scatters are hot thermal electrons, a single photon undergoes several scatterings before escaping, gaining energy with each scattering. The implication of this model is that soft photons eventually become hard photons, and that variations in a soft X-ray band should precede those in a harder X-ray band. Kaastra and Barr (1989) report that in NGC 5548 the soft X-ray variations precede the hard X-ray variations by $1-2$ hours, which would be the expected timescale associated with a Comptonization model. However, because of the uncertainties in the EXOSAT ME fluxes, it is difficult to be absolutely confident of this result. A definitive determination of a time delay between hard and soft X-ray variability is an observation of critical importance, which hopefully will be conducted with future X-ray telescopes.

\subsection{The origin of the emission from the blue bump}

The discovery that the power generally peaks in the optical to soft X-ray region implies a primacy to the underlying physical mechanism. It had long been suggested that a black hole with a rotating accretion disk lies at the heart of the AGN (e.g., review by Rees 1984), so the suggestion that the blue bump was thermal emission from the accretion disk (Shields 1978) was enthusiastically welcome. During the past decade, there has been an enormous effort to develop increasing more realistic (and complex) accretion disk emission models and to obtain the data necessary to define the continuum.

The structure and dynamics of accretion disks are governed by gravity in conjunction with thermal pressure, radiation pressure, and electromagnetic stresses. For luminosities below the Eddington luminosity, and when electromagnetic stresses may be neglected, the accretion disk has a particularly simple form, being geometrically thin but opaque (Shakura and Sunyaev 1973). Under the assumption of cylindrical symmetry and time independence, the physics governing the thin accretion disk model is rather modest. The three important parameters of the disk are the viscosity parameter $\alpha_{\mathrm{v}}$, mass flux, and the mass of the central black hole $M_{\mathrm{bh}}$. This leads to an analytic solution, from which a temperature and density is determined at each radius. The accretion disk radiates locally as a black body, and the bolometric luminosity in dominated by emission from the inner regions where the temperature is greatest. A maximum temperature is established near the last stable orbit about the black hole, which, for the vertically averaged $\alpha$-disk, is (Shakura and Sunyaev 1973):

$T=\left(4.5 \times 10^{5} K\right) \alpha_{\mathrm{v}}^{-1 / 4}\left(M_{\mathrm{bh}} / 10^{8} M_{\odot}\right)^{-1 / 4}\left[r /\left(2 G M_{\mathrm{bh}} / c^{2}\right)\right]^{-3 / 8}$.

The last stable orbit around a Schwarzschild black hole is $r=3\left(2 G M_{\mathrm{bh}} / \mathrm{c}^{2}\right)$, while for a maximally rotating black hole (Thorne and Page 1974), $r$ $=0.62\left(2 G M_{\mathrm{bh}} / \mathrm{c}^{2}\right)$. For either type of black hole, and reasonable choices for $\alpha_{\mathrm{v}}$ and $M_{\mathrm{bh}}$, the maximum temperature is $10^{5}-10^{6} \mathrm{~K}$. The emergent spectrum is the sum of the black body radiation from different radii along the disk, and if one neglects electron scattering and general relativistic effects in the radiative transfer, the emergent spectrum will have the form $F_{v} \propto v^{1 / 3}$.

This model was first applied to a complete infrared through ultraviolet spectrum by Malkan and Sargent (1982) and Malkan (1983). They assumed that 


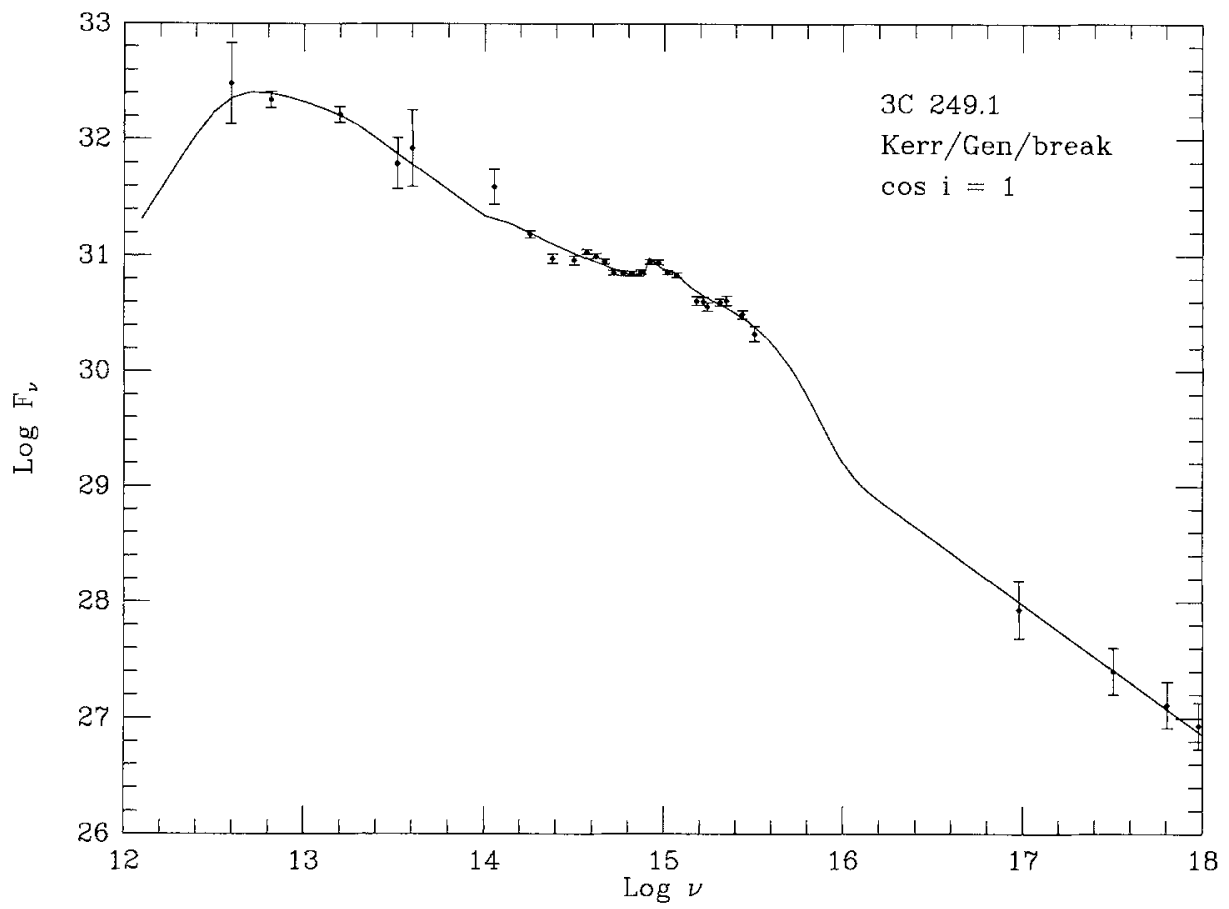

Fig. 5. The broad-band flux density $\left(F_{y}\right)$ distribution for the quasar $3 \mathrm{C} 249.1$ is compared to a model (from Sun and Malkan 1989). Like the average quasar, the peak power occurs in the ultraviolet region. The infrared through X-ray data are fit by an underlying power-law continuum with a $3000 \mathrm{~A}$ feature (Balmer continuum and Fe II emission) and the thermal emission from a thin accretion disk around a rotating black hole

in this spectral region there exists an underlying power-law spectrum whose slope and intensity may be derived from the near infrared data. Upon subtracting this power-law and correcting for Balmer continuum and Fe II emission, the resulting spectrum has a slope of about $v^{1 / 3}$, as expected for an accretion disk. The spectrum flattens near the last (highest frequency) ultraviolet continuum points, which limits the maximum temperature to be near $3 \times 10^{4} \mathrm{~K}$, slightly lower than anticipated.

The success of these initial efforts suggested that with increasingly realistic models, one might be able to extract considerably more information about the system, such as the inclination of the accretion disk, the mass of the black hole, and the luminosity relative to the Eddington luminosity. Sun and Malkan (1989) used a rotating black hole and included the effects of general relativity upon the emergent spectrum (i.e., the effect of disk inclination). This model was fit to the spectrum of $60 \mathrm{AGN}$ to determine inclination angles, black hole masses, accretion rates, and luminosities (Fig. 5). They also attempt to take into account the vertical structure of such a disk by introducing non-LTE model atmospheres to the calculation. However, this produced unacceptable fits and was not pursued (a problem also faced by Wade 1984 in a study of stellar accretion disks).

In a parallel effort, Czerny and Elvis (1987) used a nonrotating black hole and concentrated on the modification to the emergent spectrum due to Com- 
ptonization and to free-free absorption and scattering (also see Wandel and Petrosian 1988). Provided that this is the only important opacity source, scattering dominates absorption for temperatures exceeding $\approx 3 \times 10^{4} \mathrm{~K}$ (for typical accretion disk parameters). The modified black body emission flattens near $0.1\left(T / 3 \times 10^{4} \mathrm{~K}\right) \mu \mathrm{m}$, and at higher frequencies, it has a negative slope $\left(F_{v} \propto v^{-0.4}\right.$; Wandel and Petrosian 1988). This modified black body spectrum can fit the flattening (or turndown) seen in the ultraviolet, and does so through opacity effects rather than by setting a maximum temperature. However, a shortcoming of this model is that when fit to an object, the luminosity is superEddington (also, Bechtold et al. 1987), in violation of a underlying assumption of the model. One way of avoiding this super-Eddington dilemma is to employ a rotating black hole, which is more efficient at extracting energy from an accretion disk (alternative models with thick accretion disks may offer another solution).

Although the extent and shape of the blue bump in the ultraviolet and soft X-ray region are critically important for the models, they remain a matter of controversy. The first issue is whether or not the blue bump flattens or actually turns over in the ultraviolet region, a result that depends upon the continuum absorption corrections. Corrections can be made for Galactic reddening (Burstein and Heiles 1982), and for intervening absorption line systems, which depress the continuum shortward of $L \alpha$ (Steidel and Sargent 1987). Material intrinsic to the $A G N$ can be an additional important source of absorption that can further alter the spectrum. However, the presence of an extremely soft X-ray component in some sources indicates that their intrinsic absorbing columns must be less than $1 \times 10^{21} \mathrm{~cm}^{-2}$ (Turner and Pounds 1989), possibly several times less (Kruper et al. 1990). For a gas to dust ratio of 100, this would correspond to $A_{\mathrm{v}} \leq 0.1-0.2$, which is consistent with the values estimated from emission line reddening arguments and continuum reddening studies (MacAlpine 1985; MacAlpine et al. 1985; O'Brien et al. 1988a). After taking into account all corrections for absorption, some objects show a turndown in $v F_{v}$ near the low wavelength limit of the IUE (Malkan 1988, 1989), while others continue to increase (Elvis, M. 1989 for general discussion; also, Fig. 2). However, the flux densities determined from the shortest wavelength IUE spectra have significant uncertainties, and we look forward to the high accuracy ultraviolet data from the Hubble Space Telescope and ASTRO to accurately define these continua.

The second important issue is whether the big blue bump extends into the soft X-ray region. It seems natural to ascribe the steep soft X-ray excess seen in some objects $(\S 2.1)$ to the high frequency tail of the blue bump rather than to a new component. A smooth connection between the ultraviolet and X-ray regions would obviate against an ultraviolet turnover. There are examples of objects with rising ultraviolet branches and soft X-ray excesses that may represent different parts of a single blue bump. Sun and Malkan have not yet incorporated the X-ray extension in their published models, although their edge-on models do extend into the very soft X-ray region (but it may not be able to reproduce the observations). Czerny and Elvis were able to fit a bump extending into the X-ray region by using an accretion disk with a peak temperature $\sim 50$ $\times 10^{5} \mathrm{~K}$, (with or without an underlying power-law), but the luminosities are super-Eddington. Models that can fit these broad blue bumps at sub-Eddington luminosities will need to take into account both opacity effects and general 
relativistic effects. An important step in this direction has been taken by Laor et al. (1990), who have included all of these effects in their model. These promise to be the most successful thin disk models capable of reproducing the observations.

The success and attention given to the thin accretion disk models was muted by the lack of two observational expectations: large polarization and Lyman continuum absorption (e.g., Malkan 1989). In a realistic accretion disk, the gas has a vertical structure whereby the density decreases vertically until it becomes transparent. The presence of this density gradient, coupled with the hydrogen bound-free opacity should produce a visible Lyman limit absorption discontinuity (Kolykhalov and Sunyaev 1984; Laor and Netzer 1989), which is not seen (e.g., Antonucci et al. 1989). However, it is recognized that the strength of the Lyman discontinuity depends upon the surface gravity, or alternatively, the temperature gradient of the vertical structure (e.g., see discussion in Sun and Malkan 1987). A shallow temperature gradient would result in an undetectable Lyman discontinuity. Such a temperature gradient would occur if additional heating in a halo led to a wind, although for a disk without a wind (but with a low surface gravity), a detailed radiative transfer calculation shows that the Lyman discontinuity may be too small to be detected (Laor et al. 1990).

The second observational prediction of a thin disk model is that the degree of linear polarization in the continuum should usually exceed the observed values of 1-2\% (Martin et al. 1983; Stockman et al. 1984; Cheng et al. 1988; Laor and Netzer 1989). The expected polarization can be reduced if the disk is rippled (Coleman and Shields 1989), if the scale height of the atmosphere is large compared to the radial scale length of the disk, or if the disk is viewed face-on. Laor et al. (1990) showed that the polarization is naturally reduced by including the consequences of general relativity and opacity. They find that relative to the simple thin accretion disk model, the polarization at low frequencies is reduced by opacity effects while at high frequencies it is reduced by general relativistic effects. This results in a polarized flux that is only $2 \%$ for a nearly edge-on disk $\left(\theta=84^{\circ}\right.$, where $\theta$ is the angle of the rotation axis with the plane of the sky) and $\approx 1 \%$ when $\theta=60^{\circ}$, which is consistent with observation. Therefore, neither strong polarization nor strong absorption is expected from this accretion disk model, in agreement with existing observations.

\subsection{The origin of the infrared continuum}

The commonly accepted origin for the infrared emission $(1-1000 \mu \mathrm{m})$ in $\mathrm{AGN}$ has changed dramatically in the past two decades. Through the mid-1970s, the favored view was that the infrared region, like other parts of the continuum, was produced through nonthermal processes. In support of this, the radio emission $(1-100 \mathrm{GHz})$ from radio-loud objects, which was clearly nonthermal, appeared to extend smoothly into the infrared region, suggesting that it too was nonthermal. Furthermore, the near infrared continuum could be fit by a power-law, which was also consistent with a nonthermal emission mechanism. Alternatively, it was suggested as early as 1967 that thermal emission might be responsible for the infrared excess in the Seyfert 2 galaxy NGC 1068 (Pacholczyck and Wisniewiski 1967); radiation from dust was advanced as a possible mechanism (Rees et al. 1969; Penston et al. 1974). However, this appeared to 
be an unlikely alternative to the nonthermal picture, and without the general ability to obtain measurements in the submillimeter and far infrared regions, supporting evidence was not forthcoming.

A change in perspective began with infrared observations of galaxies known to be sites of active star formation. This 40-160 micron infrared emission was interpreted as thermal emission from dust heated by starlight. A similar spectrum occurs in the Sy 2 galaxy NGC 1068, which was found to have a peak flux density near 80 microns (Hildebrand et al. 1977; Telesco and Harper 1980) and had a radio-submillimeter spectrum similar to starburst galaxies (Elias et al. 1978). When the Infrared Astronomical Satellite (IRAS) was launched in 1983, it became possible to examine a greater number of $A G N$, and it was found that some were similar in appearance to NGC 1068 (Neugebauer et al. 1984; Miley et al. 1984; Miley et al. 1985). Paralleling this work were a variety of near infrared observing programs of starburst galaxies and AGN that were designed to investigate the structure of the continuum and the underlying physical processes (e.g., Neugebauer et al. 1976, 1979; Rieke 1978; Rieke et al. 1980; Balzano and Weedman 1981; Hyland and Allen 1982; Rieke et al. 1985). These authors found steeply falling near-infrared continua for Sy 2 galaxies and more moderate power-law continua for the Sy 1 galaxies. From these investigations, it was suggested that the spectra of Sy 2 galaxies was the result of emission from dust. Provided that the emission from "true" AGNs, such as Sy 1 galaxies, was nonthermal, the dust reemission picture for certain classes of objects could be accepted.

Dust in star-forming regions in galaxies often show a distinct spectral feature in moderate resolution infrared spectra. These features were searched for in the brightest Sy 1 galaxies, and found in a fraction of the objects observed (Roche et al. 1984; Jones et al. 1984; Aitken and Roche 1985). It seemed clear that at least some of the Sy 1 galaxies contained dust that was responsible for part of the near infrared emission. However, the failure to see the dust feature in all objects either means that thermal dust emission is often not an important contributor to the continuum emission, or that the response of the dust to the environment of the AGN has led to the loss of the spectral feature. Additional support for the dust model was provided by Lebofsky and Rieke (1980), who, in a work of some prescience, used the near infrared variability of AGN to argue that optical and ultraviolet emission was being reemitted in the infrared by dust grains.

As the dust hypothesis was still far from convincing, additional diagnostics were needed to discriminate between the thermal and nonthermal hypothesis. IRAS observations proved essential in providing such information. In an early study of IRAS data, Miley et al. (1985) detected 116 of $186 \mathrm{Sy}$ 1s and Sy 2s from the Veron-Cetty and Veron catalog, although this does not form a complete unbiased sample. They found the luminosity functions of the two types of Seyfert galaxies to be similar, but the mean spectra were statistically distinct. The mean Sy 1 spectrum showed that $\langle\alpha(25-60 \mu \mathrm{m})\rangle \approx\langle\alpha(60-100 \mu \mathrm{m})\rangle=-1.0$, while for the mean Sy 2 spectrum, $\langle\alpha(25-60 \mu \mathrm{m})\rangle=-1.2$ and $\langle\alpha(60-100 \mu \mathrm{m})\rangle=-0.8$. However, the dispersion in the far infrared colors for any one object is great enough that the two types of Seyfert galaxies occupy nearly the same region in an infrared color-color plot.

An important advance was realized from studies of complete samples of Seyfert galaxies, which were carried out by two independent groups. Edelson 
et al. (1987) studied the CFA sample of Seyfert galaxies, which is an unbiased, spectroscopically selected sample composed of nearly equal numbers of Type 1 and Type 2 objects. They confirmed that there was a modest difference in the spectra of the two types of objects as measured by the distribution of $\alpha(12$ $60 \mu \mathrm{m})$. From a comparison of large and small aperture 10-12 $\mu \mathrm{m}$ measurements, they found that this emission is spatially extended in all of the Sy $2 \mathrm{~s}$ and about half of the Sy $1 \mathrm{~s}$. The extended emission is attributed to emission from the disk of the galaxy on a size scale of $\mathrm{kpc}$. These extended sources are generally less luminous than the compact objects and they are spectrally distinct in that they have a cold far infrared excess. One interpretation of this result is that thermal dust emission dominates the infrared continuum in all Sy $2 \mathrm{~s}$ and half of the Sy 1 s.

A similar perspective was developed in the parallel effort of Carleton et al. (1987), in which a complete sample of hard X-ray selected Seyfert 1 galaxies were studied from the $\mathrm{X}$-ray through the radio region. Of the 34 objects in the sample, 21 have sufficient data for analysis and are not contaminated by strong emission from the host galaxy. They point out that objects with weak blue bumps have strong infrared emission, which would occur if the ultraviolet emission is absorbed and reradiated by dust. The dust would have a distribution of temperatures, with the hottest material $(1000 \mathrm{~K})$ lying $\sim 1 \mathrm{pc}$ from the central engine and cooler dust $(45 \mathrm{~K})$ at kiloparsec distances. They suggest that the emission is ultimately driven by a central non-thermal source, and that this continuum is visible in the infrared region whenever the thermal contribution from dust is weak (as in the case of the quasar 3C 273 and Sy 1 s with high ultraviolet to infrared luminosity ratios).

If dust is responsible for the infrared emission, then its spectral signature could be searched for. In particular, at long wavelengths $(100-1000 \mu \mathrm{m})$, dust does not emit as an optically thick black body, so the spectral slope can, and sometimes does exceed $\alpha=2$; this type of infrared-submillimeter spectrum is seen in some starburst galaxies (e.g., Telesco 1988). In contrast, if the infrared emission is synchrotron radiation that becomes self-absorbed in the submillimeter band, then the maximum slope should be $\alpha=2.5$. This slope would be shallower if there was not a sharp boundary to the synchrotron emitting region (Sanders et al. 1989). To test these ideas, detailed studies of the infrared through millimeter region were conducted (Chini et al. 1987, 1988; Chini et al. 1987; Antonucci and Barvainis 1988; Engargiola et al. 1988; Krugel et al. 1988; Barvainis and Antonucci 1989; Chini et al. 1989). For all radio quiet AGN, there is a sharp spectral turndown in the submillimeter region that is approximately at the same location in the rest frame of the object. For some objects, $\alpha>2.5$ in the region of the spectral turndown, inconsistent with a synchrotron picture but consistent with a dust model for the emission. Furthermore, the gas content inferred from the dust masses is consistent with the amounts observed, $\sim 10^{10} M_{\odot}$ (Barvainis et al. 1989; Krugel et al. 1989). For the radio loud objects, the origin of the infrared continuum is less clear. Some objects possess an infrared continuum that is a smooth extension of the radio-submillimeter continuum (consistent with a nonthermal origin), and other objects show an infrared component distinct from the lower frequency emission (consistent with a thermal dust origin).

A more extreme point of view was presented by McAlary and Rieke (1988) and Sanders et al. (1989), who argue that thermal emission is responsible for 
the entire infrared continuum from all AGN, with the exception of the blazar class. McAlary and Rieke (1988) presented optical and infrared continua of $16 \mathrm{Sy} 1$ galaxies with the galaxy contribution removed. From the shape of the continua and the uniformity of the near infrared colors, they argue that the emission is likely to be caused by a thermal process, most likely reemission from warm dust. Sanders et al. (1989) investigated the origin of the emission processes in the X-ray to radio spectra for the PG sample of 109 quasars, which form a complete, optically selected sample. This sample contains radio loud sources as well as radio-quiet quasars, the latter forming the majority of objects. A striking aspect of these composite energy distributions is the local minimum near $1 \mu \mathrm{m}$ (in the rest frame; $v$ vs $v F_{v}$ ) for nearly every object. This feature, which was also discussed by others (e.g., McAlary and Rieke 1988) would arise naturally from thermal dust emission, which is cutoff at wavelengths shorter than $1 \mu \mathrm{m}$ because dust is destroyed at temperatures exceeding $2000 \mathrm{~K}$. Sanders et al. (1989) point out that this $1 \mu \mathrm{m}$ feature is strong in radio-loud objects as well, leading them to conclude that most of the infrared emission in these objects is due to thermal dust emission.

The shape of the infrared emission cannot be reproduced by dust at a single temperature, but by dust with a range of temperatures. For dust emission models, it is assumed that the temperature of the dust is established by a balance between the energy absorbed and emitted, so that the hotter dust lies closer to the central radiation source (Barvainis 1987; Phinney 1990). In particular, the radius of the hottest infrared emitting region $(1000-2000 \mathrm{~K})$ would be $\sim 0.3-1 \mathrm{pc}$ at $1-2 \mu \mathrm{m}$ while the radius of the coolest regions $\left(30-60 \mathrm{~K}\right.$ ) would be $\sim 10^{3}-10^{4} \mathrm{pc}$ at $60-100 \mu \mathrm{m}$ (Edelson et al. 1988 find that at least half of the $155 \mu \mathrm{m}$ from NGC 4151 is due to dust emission in an extended region). The shape of the infrared emission determines the size (surface area) of the dust region as a function of temperature (equivalently, the solid angle subtended by the dust as a function of radius). To reproduce the observations of a typical object, this solid angle must increase with radius, which would occur in a warped disk (Phinney 1990). A qualitative prediction of these models is that infrared variability, should it arise, would occur on a timescale of months-years at 1-2 $\mu \mathrm{m}$ and thousands of years at $100 \mu \mathrm{m}$. The lack of variability in the IRAS bands supports this prediction (Clement et al. 1987; Edelson and Malkan 1987) as does the decrease in the variability of most objects between the optical and infrared regions (Cutri et al. 1985). Furthermore, Clavel et al. (1989) find a 400 day delay between $3.5 \mu \mathrm{m}$ and 1-2.2 $\mu \mathrm{m}$ emission in Fairall 9, which may be interpreted as due to thermal emission from dust at a distance of $\sim 11 \mathrm{tt}-\mathrm{yr}$.

While there is strong evidence that the infrared continuum in about half of the Sy $1 \mathrm{~s}$ and quasars has a thermal origin, there is still a controversy as to whether this applies to the near infrared continuum from all such objects. In support of the nonthermal infrared model is the lack of the dust feature in 2-11 $\mu \mathrm{m}$ spectra of some objects, the apparent power-law infrared-optical continuum in a minority of sources, and the statistical connection between the infrared and the X-ray continuum. Regarding this last point, the soft X-ray flux density correlates better with the infrared emission than with emission from other wavelength bands according to Edelson and Malkan (1986) and Carleton et al. (1987). Furthermore, the near infrared and X-ray slopes are similar $(\alpha(1.25-$ $2.2 \mu \mathrm{m}) \approx \alpha(0.5-4 \mathrm{keV})$ ), and if the near infrared continuum were extended to $\mathrm{X}$-ray energies, it would pass through the $\mathrm{X}$-ray flux density (to within the 
considerable uncertainties). This finding suggests that there is an underlying nonthermal power-law continuum that is responsible for both the near infrared and the X-ray emission. However, doubt is cast upon this result by Sanders et al. (1989), who, using a larger sample, fail to confirm this finding. Also, narrow aperture near infrared photometry, which minimizes the galaxy contribution, indicates that the infrared slope can be considerably steeper than the X-ray slope (Rieke 1985; Simons et al. 1988; McAlary and Rieke 1988), inconsistent with a continuous infrared through $\mathrm{X}$-ray power-law continuum. Furthermore, the presence of an apparent power-law infrared-optical continuum and the lack of the spectral dust feature would be consistent with, but not proof of nonthermal emission.

This author is of the opinion that the infrared continuum in nearly all radio quiet AGN has a thermal origin. The submillimeter turnover seen in AGN is similar to that seen in starburst galaxies, it occurs at approximately the same rest wavelength in all objects, and is easily understood by the rapidly decreasing emissivity function of dust in this waveband. The second strong piece of evidence supporting the dust model is the dip in the power per logarithmic bandwidth, which occurs at a rest wavelength corresponding to the condensation temperature of dust $(\approx 1-2 \mu \mathrm{m})$. A prediction of the thermal model is that the size of the emitting region is greater than a light year, while for the synchrotron model, the emitting region is a small fraction of a light year. The lack of variability at wavelengths longer than $2 \mu \mathrm{m}$ in radio quiet sources offers further support for the thermal dust model.

For some radio loud objects, nonthermal emission can make a measurable contribution to the infrared continuum. For example, 3C 273 shows infrared variability on a timescale of days to weeks (Gear et al. 1983; Cutri et al. 1985; Marscher and Gear 1985; Courvoisier et al. 1987, 1988, 1990), implying a component with a brightness temperature $>10^{6} \mathrm{~K}$, which most probably has a nonthermal origin. As will be discussed below ( $\$ 2.6, \S 3.7)$, many of the radio-loud objects may be a combination of blazar and radio-quiet quasar, thereby harboring both a thermal and nonthermal emission component in the infrared region.

\subsection{Dust imbedded AGN or starburst galaxies}

Both starburst galaxies and AGN can be strong sources of infrared emission, raising the issue of whether there might be a relationship between these sources. A fundamental question that has been examined by many investigators is whether the source of the infrared luminosity is primarily star formation (a starburst galaxy) or reradiated emission from the central engine in an AGN. Secondly, is there evolution from a starburst galaxy to an AGN or from a gas-rich to a gas-poor AGN?

By obtaining observations in various wavebands, it is now possible to identify whether or not an AGN is present within an infrared luminous galaxy. First, it is important to realize that galaxy orientation is unlikely to affect infrared brightness. A galaxy should be nearly transparent to $60 \mu \mathrm{m}$ emission, as it would require $\mathrm{N}_{\mathrm{H}} \sim 10^{24} \mathrm{~cm}^{-2}$ for dust to become an important absorber at these wavelengths (after Draine and Anderson 1985); this column is greater than that for an edge-on galaxy. The low opacity, combined with the isotropic nature of dust emission make the $60-100 \mu \mathrm{m}$ fluxes an excellent indicator of infrared 
bright objects, regardless of the orientation of the galaxy. In order to differentiate between an AGN and a starburst galaxy, it is necessary to find other observational tracers that are different in the two types of systems and whose detection is independent of galaxy orientation.

Radio emission can provide this type of tracer, if the dust and gas does not completely quench the production of the radiation. Fortunately, radio emission can be detected from all Seyfert galaxies in a properly chosen volume-limited sample (Ulvestad and Wilson 1984; Meurs and Wilson 1984; review by Wilson and Heckman 1985), and from starburst galaxies with nearly equal ease (Norris et al. 1988; Wilson and Heckman 1985). The radio morphology of a starburst galaxy is characterized by diffuse disk-like emission (scale $>0.2 \mathrm{kpc}$ ), while in a Seyfert galaxy it is composed of a core (scale $<10 \mathrm{pc}$ ), jets, and lobes (Wilson and Heckman 1985; Wilson 1988). In low power sources such as these, the jet and lobe emission is isotropic (the lobes and jets on both sides are about the same brightness), but the core may emit anisotropically due to beaming (e.g., review by Bridle 1987). Nearly all infrared bright sources chosen for study have been detected in the GHz range with the VLA (e.g., Norris et al. 1988), and even in a complete sample around the south galactic pole (which included many low luminosity objects), 131/154 were detected with a flux density above $1 \mathrm{mJy}$ (Wolstencroft et al. 1988). Evidently, despite the abundant gas and dust in these galaxies, the radio emission is still detectable. If the dust and gas were to quench the radio emission emerging from the central engine of an AGN, there would be a population of IRAS bright galaxies that are relatively radio poor; such a population does not exist.

A statistically complete sample of extremely luminous far-infrared galaxies was studied at radio wavelengths by Norris et al. (1988), Norris et al. (1988), and Wilson (1988). We note that to distinguish between a small starburst event and an AGN core, the objects being studied should be close enough that the radio interferometer can resolve the central starforming region, which is typically $0.2 \mathrm{kpc}$ in size; this observational condition is not always met. From the radio maps, these investigators were able to identify some objects as starburst galaxies and some as Seyfert galaxies. For the diffuse sources (starburst galaxies), the radio to infrared ratio is more tightly defined than for Seyfert galaxies, which are relatively more luminous radio sources and with a broader dispersion. The high resolution Parkes-Tidbinbilla interferometer $\left(0.1^{\prime \prime}\right.$ resolution) was used to search for the compact cores expected from Seyfert galaxies (Norris et al. 1988). In one case, 0/9 starburst galaxies and 5/19 Seyfert galaxies were detected while in another sample, $0 / 43$ starburst galaxies and 3/9 Seyfert galaxies were detected. At radio wavelengths, Seyfert galaxies appear to be distinct from starburst galaxies even in gas and dust rich systems.

Hard X-ray emission can also be used to discriminate between AGNs and starburst galaxies, since the former are much stronger sources of this radiation. The magnitude of the X-ray flux, which is thought to be produced in a small region near the central engine, should not be influenced by obscuring gas and dust in the galaxy. The optical depth to photons whose energies exceed a few $\mathrm{keV}$ is the same or less than that for $60 \mu \mathrm{m}$ photons, so the galaxy is transparent to hard X-rays. Rieke (1988) examined 15 ultraluminous infrared galaxies detected by IRAS $\left(L_{\mathrm{ir}}>1 \times 10^{11} L_{\odot}\right)$ for which good X-ray data is available from the HEAO-1 A 1 experiment (2-10 keV energy band). One object was detected (NGC 7469, a Seyfert galaxy) and the $3 \sigma$ detection of another source 
is attributed to source confusion. The upper limits are used to show that for the observed bolometric luminosity, the X-ray luminosity is one to two orders of magnitude below the value expected for an AGN. They argue that the bulk of the infrared luminosity in these objects is generated by intense circumnuclear star formation rather than by reemission of $\mathrm{AGN}$ radiation.

Another indication that AGNs are not completely hidden by the gas and dust is the presance of high excitation optical emission lines that characterize Sy 1 and Sy 2 spentra. Wilson (1988) finds that when the radio structure is indicative of the S:yfert phenomenon, high ionization emission lines are present, even in the IRAS-bright objects (conversely, starburst-type radio structures have low ionization emission lines). Because the radio emission is able to emerge from the center, and Seyfert galaxies often produce extended optical emission line structures associated with the radio jets and lobes (Unger et al. 1987; Wilson et al. 1988; Haniff et al. 1988; Pogge 1988; Shields and Filippenko 1988), it is not surprising that an emission line signature is evident. This implies that the ionizing field is anisotropic, which Netzer $(1985,1987)$ has also argued for, based on the properties of the broad emission lines. The association of the narrow high excitation lines with the radio emission typical of AGN indicates that the presence of the dust and gas responsible for the IRAS emission does not restrict the visibility of the narrow high excitation lines. The dust may be an agent in obscuring the broad emission line region, since the ratio of Sy $1 \mathrm{~s}$ to Sy $2 \mathrm{~s}$ is lower in the IRAS selected sample $(1: 2$; Lawrence 1988) than in an optically selected sample (1:1).

To summarize, the evidence suggests that few dust obscured AGN masquerade as starburst galaxies. Objects such as Arp 220, NGC 6240 and NGC 4945 may hide AGN, although this is a controversial, and even if true, these sources would be the exception rather than the rule (Wright et al. 1984; Soifer et al. 1984; Brock et al. 1988; Eales and Arnaud 1988; Norris et al. 1988; Smith et al. 1989). Sources without an AGN signature derive most of their infrared emission from the starburst phenomenon. Infrared bright galaxies with an AGN signature often have two sources for the infrared emission: star formation, and reradiated emission from the AGN (e.g., Edelson et al. 1987; Soifer et al. 1987).

The distribution of infrared-bright galaxies into starburst galaxy and AGN is a strong function of infrared luminosity. The very highest infrared luminosities are nearly all associated with AGN, as shown by Sanders et al. (1988a), who found that $9 / 10$ galaxies with $L(8-1000 \mu m)>1 \times 10^{12} L_{\odot}$ had optical AGN spectra. With a somewhat different sample and different selection criteria, Lawrence (1988) also finds that most objects show AGN characteristics when $L(60 \mu \mathrm{m})$ $>1 \times 10^{12} L_{\odot}$, but that when $1 \times 10^{12} L_{\odot}>L(60 \mu \mathrm{m})>1 \times 10^{11} L_{\odot}$, few show AGN properties, suggesting that most are starburst systems. Weak starburst systems and ordinary galaxies are the most common sources at luminosities $<1 \times 10^{11} L_{\odot}$ (Lawrence 1988).

Another important issue is whether there is evolution, either from a starburst galaxy to an $\mathrm{AGN}$, or from a gas and dust rich $\mathrm{AGN}$ to a normal $\mathrm{AGN}$ (Sanders et al. 1988a, b; Weedman 1983, 1988). It seems unlikely that starburst galaxies without a central engine evolve into AGN since this would require the formation of a supermassive black hole (presumably from an extremely compact and dense star cluster) where one has not previously existed. It hardly seems possible for the distributed star formation present in a starburst system. to produce a central supermassive black hole. The other evolutionary suggestion, 
that a gas-rich IRAS-bright AGN evolves into an AGN of normal infrared and gas properties, is both possible and likely. There is good evidence for elevated rates of star formation in many of the IRAS-bright AGN (e.g., Wilson 1988; Lawrence et al. 1989), which should eventually consume the gas, rendering the galaxy more gas-free. If this occurs for all $A G N$, then their lifetime, relative to starburst galaxies, would be the ratio of their space densities, which is about 50 (Lawrence 1988).

\subsection{Directionality and relativistic beaming}

It is possible to "unify" classes of $A G N$ by postulating that the orientation of an AGN can lead to distinctively different appearances or classifications. This unification, which significantly reduces the number of different types of $A G N$, requires that either directionality or both directionality and relativistic beaming be important. By directionality, it is meant that the observational appearance of a source depends upon its orientation. This could arise because an obscuring torus or cone prevents one from seeing the core region except for favorable orientations. Such effects can arise without the aid of relativistic motion. Relativistic beaming is a special type of directionality due to the amplification of the observed brightness along the direction of motion. The AGN phenomenon provides examples of directionality with relativistic and nonrelativistic causes.

We infer directionality of a nonrelativistic nature plays an important role in the distinction between Sy 1 and Sy 2 galaxies. Although only narrow emission lines are seen in unpolarized light in the nucleus of Sy 2 galaxies, the polarized light constitutes a spectrum with broad emission lines, similar to a Sy 1 spectrum (Miller and Antonucci 1983; Antonucci and Miller 1985; Antonucci et al. 1987; Goodrich and Miller 1988; Thompson and Martin 1988; Goodrich 1989). Also, the position angles are approximately perpendicular to the axis of the radio structure. The picture that has been developed to explain this phenomena places the nuclear region of the AGN (out to at least the radius of the broad emission line region, about $0.1-1 \mathrm{pc}$ from the center) within an opaque cone or torus with an opening angle of $\sim 60^{\circ}$ (above authors; also Krolik and Begelman 1986, 1988). Unless one's line of sight lies within the opening angle of the cone, the core region is not directly visible. Should the inner surface of the cone or torus be hot and ionized such that electron scattering is important, we should be able to see over the near side of the cone and observe the broad emission line radiation scattered off the far side; this would lead to faint, polarized broad emission lines, as observed. While there are still issues to be resolved regarding the scattering process, it is possible to unify the two types of Seyfert galaxy into one phenomenon, and explain the polarization measurements.

Much of the literature dealing with the unification of classes of sources have focused upon radio-loud objects. Scheuer and Readhead (1979) proposed the first detailed unification model, which brought together radio-loud and radio-quiet quasars. Subsequent evidence revealed serious problems with this model (e.g., Bridle and Perley 1984), thereby calling for a modification of the original idea. A less ambitious model was proposed by Orr and Browne (1982), who argued for a unification of core-dominated radio-loud quasars with lobedominated quasi-stellar radio sources. Since this work, a wealth of observations 
have been accumulated that permit a proper statistical examination of the predictions of this model. Barthel (1989) has used the data available for the $3 \mathrm{CR}$ sample to refine the model and build a convincing case for the unification of luminous radio galaxies and the radio-loud quasars (for objects where $L_{\text {radio }}$ $>10^{26} \mathrm{~W} \mathrm{~Hz}^{-1}$; also known as FRII sources). Relative to the radio-loud quasars, the radio galaxies are narrow-lined, have weaker radio cores and jets (Miley 1980; Owen 1986), and the extended radio structure is larger. The dependence of the size of the extended radio sizes with object type can be explained if the radio galaxies are closer to the plane of the sky (mean angle to the line of sight of $69^{\circ}$ ) than the radio-loud quasars (mean angle to the line of sight of $31^{\circ}$; Barthel 1989). Differences in the intensity and polarization of the emission lines are parallel to the Sy 1/Sy 2 case, and line polarization studies support this analogy (Antonucci 1984). As in the case of the Seyfert galaxies, an obscuring torus is needed near the nucleus. If free electrons are plentiful so that the radio free-free absorption is high, this obscuring torus might be opaque to the radio core as well. However, relativistic effects (beaming) need to be added in order to explain the dominance of the jet on only one side of the center in luminous radio galaxies. The side of the radio source with the jet has the less depolarized lobe, which would place it closest to us, as expected if relativistic beaming is present (Laing 1988; Garrinton et al. 1988; for a complementary approach using optical emission lines, see Axon et al. 1989). Relativistic beaming, rather than an obscuring torus may be responsible for the apparent brightness of the radio cores. Therefore, unification of these objects require both obscuration of the emission lines (non-relativistic directionality) and beaming of the radio emission (relativistic beaming).

Since there is no reason to expect an obscuring torus and relativistic beaming to have the same opening angle, it is possible to have one quasar component visible (broad lines) while the other remains undetected (core radio emission). Barthel (1989) suggests that this intermediate case leads to a third type of AGN, the broad-line radio galaxy, which have strong broad emission lines, a weak radio core, and luminous extended radio emission. This picture requires that the radio continuum emission is beamed into a more narrow cone than the angle defined by the opaque cone or torus obscuring the broad emission lines. In fact, the opening angle of radio jets as defined by kiloparsec scale radio maps (e.g., Bridle 1987) is less than the opening angle of the obscuring cone estimated from the fraction of sources with broad emission lines. Consequently, this model unifies three classes of objects with luminous extended radio emission: narrow line radio galaxies, broad line radio galaxies, and radio-loud core-dominated quasars. The unification of blazars with their less exotic AGN counterparts will be discussed in $(\$ 3.7)$.

In the first of two objections to this unification, Prestage and Peacock (1988) mention that the environments of the compact and extended radio sources are different. However, the difference between the galaxy density around FRIIs and compact radio objects has a low statistical significance. Secondly, Hutchings et al. (1989) find a modest difference in the radial scale length of the underlying galaxy, the separation from companions, and the frequency of interaction. These differences depend on accurate measurements of low surface brightness features. Despite the care of the investigators, the presence of unexpectedly broad, lowlevel wings from the optically bright nucleus could be responsible for several of these results. In contrast, Smith and Heckman (1990), who have studied equal- 
ly powerful radio galaxies and radio quasars, find a strong similarity in the environments of these two classes. In the opinion of the author, the evidence is consistent with the basic unification picture of luminous radio sources, although additional statistical studies will be needed to verify (and probably clarify) this model.

\section{The blazar class}

A fundamental difference between radio-quiet quasars and blazars is that in the former group, the far infrared through ultraviolet emission is largely due to thermal processes, while in the latter, it is largely nonthermal emission. Well before this distinction was understood, an operational difference between the two classes was established based upon polarization and variability properties. An active galactic nucleus is defined as a blazar (e.g., Miller 1989) if it has a (1) smooth continuum IR-optical-UV emission from a stellar-like nucleus, (2) high optical polarization $(p>3 \%)$, (3) rapid optical variability on a time scale of days or less, and (4) a strong and variable radio continuum. When emission lines from a blazar are unusually weak (as measured by their equivalent widths), the source is classificed as a BL Lacertae object. The optically violent variable quasars (OVVs) are blazars whose emission line equivalent widths are comparable to normal radio-quiet quasars, except during periods of intense continuum flares. To investigate the continuum emission properties of blazars, the BL Lacertae objects are simpler objects for study, being uncluttered by emission lines and their related features (e.g., blue bump; we generally avoid discussions of "crossover" objects, which have properties of both an ordinary quasar and a blazar, the most famous case being $3 \mathrm{C} 273$ ).

The purpose of this part of the review is to' synthesize the observational and theoretical investigations that relate to the origin of the continuum emission. A central issue of discussion is the extent to which the jet model can describe the observations. In particular, we examine the evidence for a jet, the inhomogeneity of the emitting plasma, the radiation mechanisms within the plasma, the origin of the X-ray emission, the relativistic bulk motion of the plasma, and the evidence for a parent population. As a general resource of additional background information, the reader is referred to a recent meeting on BL Lacertae objects ("BL Lac Objects", ed. L. Maraschi, T. Maccacaro, and M.-H. Ulrich). In addition, several aspects of the radio properties of these objects are thoroughly reviewed within the works of Altschuler (1989), Mutel (1990), and Bridle (1987).

\subsection{Basic continuum properties of blazars}

A mutifrequency spectrum of a typical BL Lacertae object displays a flat radio spectrum (defined as $-0.5<\alpha<0.5$ where $F_{v} \propto v^{\alpha}$ ) that contains only a fraction of the total power (e.g., Impey and Neugebauer 1988; Makino 1990). When plotted as power per logarithmic bandwidth $\left(v F_{v}\right.$ vs $v$; Fig. 1, 6), the continuum rises smoothly from the radio into the millimeter, submillimeter, and infrared regions. For the objects shown in Fig. 6, the peak in the power distribution occurs in the submillimeter to near infrared region, although several X-ray 


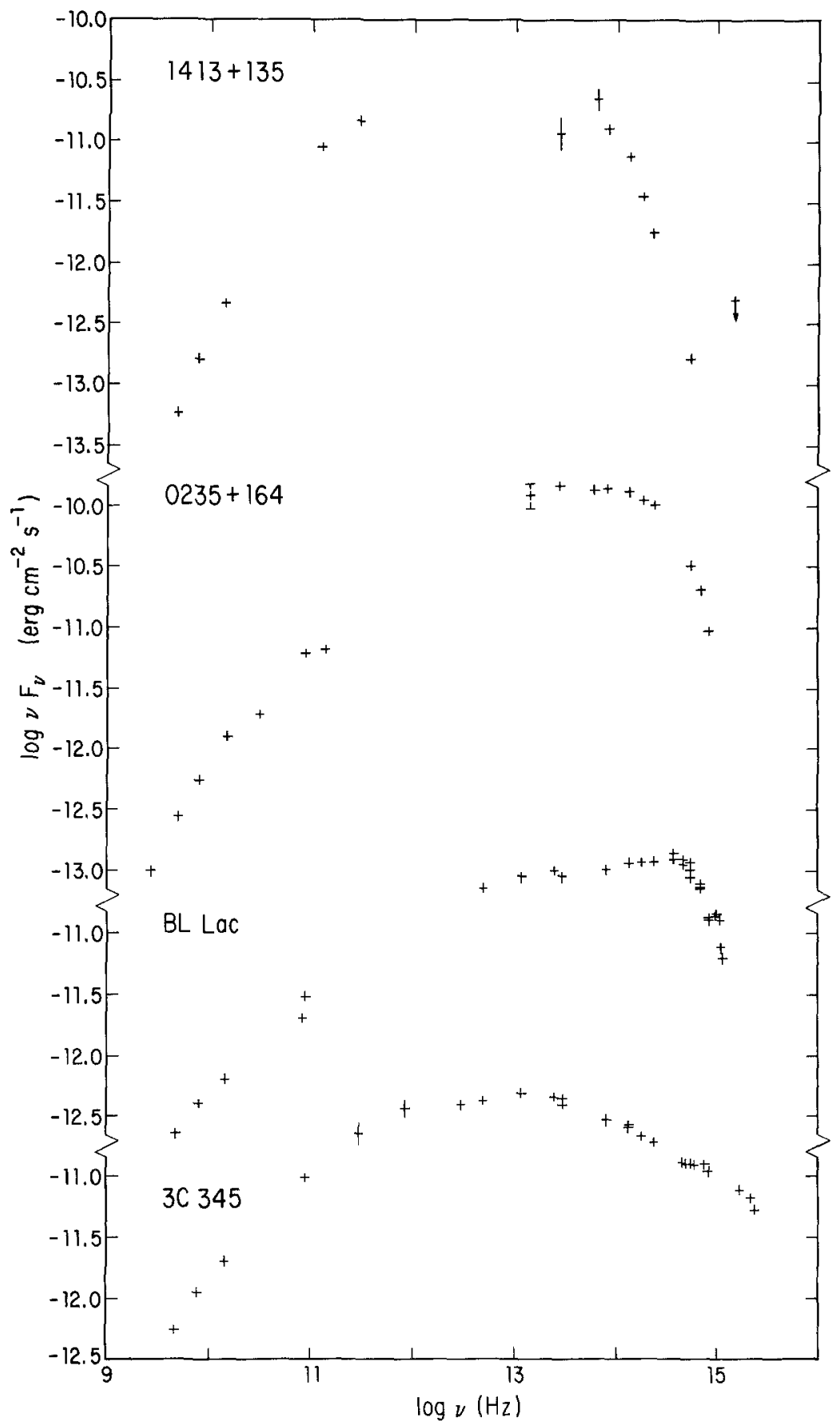

Fig. 6. The multifrequency spectrum of four blazars (Bregman et al. 1990). In these objects, most of the the power per logarithmic frequency is emitted in the $10^{11}-10^{15} \mathrm{~Hz}$, even when including the soft X-ray contribution. The sharp spectral turnover seen in the infrared-ultraviolet region for the top three objects is probably due to a high energy cutoff in the electron distribution 
selected objects have their peak power in the optical or X-ray region (e.g., Mrk 421; Makino et al. 1987). The power declines slowly at frequencies beyond the peak frequencies (typically in the infrared; Fig. 1), although the decline may be rapid and the peak power may be sharply defined if a high frequency spectral cutoff is present (Fig. 6). The power contained in the soft X-ray band is typically $\sim 10 \%$ of the total observed power, although if $\alpha_{x}>-1$, the power rises with increasing energy and the contribution to the power may be more substantial at higher energies.

Identification of continuum features can be critically important in determining the underlying radiation mechanism. In the radio quiet $\mathrm{AGN}$, features such as the $100 \mu \mathrm{m}$ falloff, the $1 \mu \mathrm{m}$ dip, and the ultraviolet peak of the blue bump, occurred at approximately the same rest frame wavelengths, thereby implying that thermal or atomic processes are important. In contrast, BL Lacertae objects have fewer spectral features, which do not occur at constant wavelength. For example, a distinctive high frequency turnover is seen in several weak-lined blazars (Beichman et al. $1981 \mathrm{a}$, b; Bregman et al. 1981; Rieke et al. 1979; Rieke et al. 1982), but the location of this feature occurs over a very broad range. This turnover is found to occur in the infrared $(1413+135)$, optical (AO $0235+164$ ), and the ultraviolet (BL Lac; Bregman et al. 1990). Another spectral feature, the peak power per logarithmic bandwidth, can occur in the submillimeter, infrared, optical, ultraviolet, or X-ray energies (Gear et al. 1985; Landau et al. 1986; Impey and Neugebauer 1988; Worrall 1989; Brown et al. 1989a, b; Makino 1990), although it most commonly occurs in the infrared region. Relationships have been found that connect various continuum properties. When the continuum resembles a parabola (in $\log v F_{v}-\log v$ space), a correlation is found between the widths of the parabola and the bolometric luminosity (Landau et al. 1986). However, the implications of the results have remained obscure and do not obviously support a thermal origin for the gas. To conclude, there are no spectral features in BL Lacertae objects that suggest a thermal origin for the emission.

There is strong evidence that the continuum emission from BL Lacertae objects is nonthermal. The radio continuum emission emerges from a region in which the brightness temperature is $\sim 10^{12} \mathrm{~K}$, which clearly implies a nonthermal process (e.g., Mutel 1990). The synchrotron process is suggested because the necessary magnetic field is inferred from the high polarization of the radio continuum. Because the radio continuum extends smoothly into the millimeter, infrared, optical, and ultraviolet wavebands, it is inferred that emission from these regions also arise from the nonthermal synchrotron process. The brightness temperatures in the infrared and optical regions, inferred from variability measurements, also support the nonthermal hypothesis. Infrared variability has been seen in the blazars OJ 287 and 3 C 345 with a typical doubling time of 2 months, which implies a $60-100 \mu \mathrm{m}$ brightness temperature $>10^{6} \mathrm{~K}$ (Edelson and Malkan 1987). This brightness temperature cannot be associated with blackbody emission processes, which has a characteristic temperature of 30-60 K at these wavelengths. Similarly, rapid optical variability (Moore et al. 1982; Miller and Carini 1989; Carini 1990) implies a brightness temperature $>10^{6} \mathrm{~K}$, also in excess of the expected thermal blackbody temperature in this waveband, $10^{3}-10^{4} \mathrm{~K}$. Although it is impossible to reproduce these measurements with thermal blackbody process, a nonthermal mechanism could be responsible for the emission. 


\subsection{Evidence for a jet}

Various observations support a picture where much of the radio through optical emission originates in an inhomogeneous plasma jet through which shocks propagate. A jet-like geometry on the parsec scale is suggested by the motion of the VLBI radio components, which usually exhibit superluminal motion (e.g., Biretta et al. 1986; Walker et al. 1987). Superluminal motion of discrete components is usually interpreted as a relativistic aberration effect of material moving with Lorentz factors of bulk motion of a few in BL Lacertae objects (e.g., Begelman et al. 1984; Cohen 1989). The opening angle of the beamed radiation can be greater than the angle defined by the bulk motion alone, a situation that can occur, for example, if non-planar shocks are manifest in the jet (e.g., Blandford 1987); opening angles of $\sim 30^{\circ}$ are implied. These superluminal knots of emission are polarized (Roberts et al. 1987; Cawthorne and Wardle 1988; Gabuzda et al. 1989) with the position angle along the direction of the jet, implying that the magnetic field is perpendicular to the jet. Radio flux monitoring observations of the entire core/jet region also have been discussed in terms of a jet model. These studies show flux density outbursts with corresponding variations in the angle and degree of polarization (Aller et al. 1985) that can be understood in a model where a shock propagates down a jet of plasma, compressing high density parcels of plasma and altering their emission properties (Marscher and Gear 1985; Hughes et al. 1989a, b). The shock compresses the plasma, preferentially raising the component of the magnetic field component parallel to the shock front, and imparts a velocity to the plasma that results in significant relativistic boosting. Since one expects the mean velocity of the shock to be along the jet axis, the magnetic field will be oriented approximately perpendicular to the jet axis, a result seen directly in the VLBI observations. One of the first applications of this type of model was to 3C 273, in which discrete variability events were followed from the optical to radio regions (Gear et al. 1983, 1986; Nolt et al. 1983; Marscher and Gear 1985; Robson et al. 1983, 1986). A shock wave passing through an adiabatic, conical, relativistic jet not only reproduces this broad-band variability, but also naturally leads to relatively flat radio spectra.

Optical polarization measurements further support this model. The optical polarization is generally large ( $p>3 \%$ by definition; but it can sometimes exceed $40 \%$, e.g., Mead et al. 1990) and variable in both degree of polarization and position angle (e.g., Moore and Stockman 1981, 1984; Brindle et al. 1985; Sitko et al. 1985; Impey 1987; Kulshretha et al. 1987; Smith et al. 1987, 1988; Mead et al. 1988, 1990; Valtaoja et al. 1989; Kikuchi 1989; Ballard et al. 1990). Although the position angle of polarization can vary considerably, it often has a preferred value - a mean or most frequently observed angle. This preferred position angle is well-correlated with the VLBI structural axis (Rusk and Seaquist 1985; Impey 1987; Smith et al. 1986), again implying a magnetic field perpendicular to the jet axis for the much smaller optical region. In summary, several lines of evidence support a qualitative "shock-in-jet" model, which appears to have validity from the optical through the radio region.

\subsection{Inhomogeneity of the jet}

Observations indicate that the emitting plasma is not uniformly distributed within the jet, and that the size of the emitting region in blazars appears to increase 
with decreasing photon energy. Although sizes can be determined directly from VLBI radio observations in the radio region, they must be inferred from variability data in the infrared, optical, and X-ray wavebands. The VLBI observations show that the diameter of spatially resolved knots and the separation of the knots from the core is typically several light years (e.g., review by Kellermann and Pauliny-Toth 1981). The core is frequently unresolved, indicating radio structures smaller than a light year in the most compact of objects (e.g., Gabuzda et al. 1989).

Size information deduced from variability data is a bit more complicated because the flux variations can occur over a variety of timescales (e.g., Fiedler et al. 1987; Bregman et al. 1988). We assume that rapid fluctuations are the most likely to contain information about the size of the source, while slower variations are likely to be dominated by radiative cooling or heating, or slow changes in the structure of the system. One can define a characteristic variability timescale $t_{\text {char }}=d t / d \ln F_{v}$ for the most rapid variations that show an amplitude in the range $0.1<\Delta F_{v} / F_{v}<1$. In practice, $t_{\text {char }}$ is insensitive to the chosen sampling interval $\Delta t$ because the amplitude of variation is less on shorter timescales (i.e., $d \ln F_{v}=\Delta F_{v} / F_{v} \propto \Delta t^{m}$, where $m=0.5-1$ so that $t_{\text {char }}$ depends weakly on $\Delta t$, if at all; after Bregman and Hufnagel 1989). Characteristic sizes are expressed as $c t_{\text {char }} \delta^{-1}$, where $\delta$ is the correction for time dilation due to the Doppler boosting. Also, the correction to the observed flux from relativistic boosting is determined by $\delta$, which has the form $\delta=[\gamma(1-\beta \cos \theta)]^{-1}$, where $\theta$ is the angle with respect to the line of sight.

Characteristic variability timescales can be determined from the radio through the X-ray region. The characteristic timescale of radio flux variation is approximately years in the $10^{8}-10^{9} \mathrm{~Hz}$ region, months-years in the $10^{9}-10^{10} \mathrm{~Hz}$ region, and weeks-months in the $0.4-1 \times 10^{11} \mathrm{~Hz}$ region (Flett and Henderson 1981; Altschuler 1982, 1983; Epstein et al. 1982; Balonek 1982; Johnston et al. 1984; Aller et al. 1985; Botti and Abraham 1987; Fiedler et al. 1987; Pustil'nik and Aliakberov 1987; Terasranta et al. 1987; O'Dell 1988; Valtaoja et al. 1988; see review by Altschuler 1989); cm radio source variability of an intrinsic nature should not to be confused with the daily variations, which are likely to have an extrinsic origin due to scintillation by interstellar clouds (Heeschen et al. 1987). Far infrared flux variations, as defined by the relatively small number of pointed IRAS observations, are found to exist for only a few blazars; the timescale is several weeks (Impey and Neugebauer 1988). In the near infrared region $(1-10 \mu \mathrm{m})$, rapid variations occur on a timescale of days (Rieke et al. 1976; Rieke and Lebofsky 1979; Robson et al. 1986; Bregman et al. 1986, 1988; Smith et al. 1987; Lorenzetti et al. 1987; Courvoisier et al. 1988; longer timescale variability is discussed by Cutri et al. 1985 and Neugebauer et al. 1989). Relative to near infrared variations, slightly more rapid variations occur in the optical waveband, where the characteristic timescale is typically a day in the best-studied sources such as BL Lac, OJ 287, AO 0235+164, and others (the range is hours to days; Rieke et al. 1976; Pica et al. 1980; Moore et al. 1982; Babadyhanzants and Belokon' 1984, 1986; Lloyd 1984; Sitko et al. 1985; Moles et al. 1986; Hagen-Thorn et al. 1987; Moore et al. 1987; Smith et al. 1987; Barbieri et al. 1988; Corso et al. 1988; Kidger 1988; Kikuchi et al. 1988; Pica et al. 1988; Sillanpaa et al. 1988; Perez et al. 1989; Miller and Carini 1989; Carini 1990; Solomos et al. 1990; Webb 1990). There is little data suitable for studying the short-term ultraviolet variability, although it does appear to 
be comparable to the optical fluctuations (Glassgold et al. 1983; Ulrich et al. 1988; Urry et al. 1988; O'Brien et al. 1988b; Celotti et al. 1989). Soft X-ray variability has been the subject of intense study, with variations as rapid as one minute in the remarkable case of $\mathrm{H} 0323+022$ (Doxsey et al. 1983; Feigelson et al. 1986; Schwartz and Madejski 1987), and variations of 1-8 hours in PKS 0548 - 322, PKS 2155-304, OJ 287, and other objects (Morini et al. 1986; Giommi et al. 1986, 1987, 1990; Agrawal et al. 1987; Barstow and Stanger 1988; Branduardi-Raymont and Mittaz 1988; Turner and Pounds 1988; Makino et al. 1989; Ohashi et al. 1989; Tagliaferri et al. 1989; Treves et al. 1989; Garilli and Macagni 1990). The $2-10 \mathrm{keV}$ continuum often displays greater variation than the softer X-ray continuum (typically 0.1-2 keV; Agrawal et al. 1987; Griffiths et al. 1989; Giommi et al. 1990). At higher energies (10-100 keV), the hard X-ray emission is known to vary, but there is little information on the characteristic timescale, except that it is years or less; Bezler et al. 1988); no variability information exists at $\gamma$-ray energies.

The decreasing value of $t_{\text {char }}$ with progressively higher energies can be characterized approximately as $t_{\text {char }} \propto v^{-\frac{1}{2}}$. In obtaining this, we have combined $t_{\text {char }}$ as determined from a variety of different objects, although this relationship has been seen for an individual object, 3C 446 (Bregman et al. 1988; optical to X-ray variability timescales for four objects are discussed by Celotti et al. 1989). While much additional information can be extracted from the variability data, this simple relationship can be a powerful constraint in developing a spatial model for the distribution of the plasma in the jet.

Another aspect of the continuum variations is whether there is a causal connection between the fluctuations seen at different wavebands. This can be tested by examining whether the variations in one waveband are correlated with those in another. We begin such comparisons in the optical region and progress to longer wavelengths. When comparing the optical and near infrared wavebands, the flux variations occur together in time and with a time delay no greater than a day, if at all (e.g., Bregman et al. 1990). A time delay of days has been seen between optical and $10 \mu \mathrm{m}$ observations, while a time delay of weeks is found when comparing optical and far infrared IRAS measurements (Marscher and Gear 1985; Robson et al. 1986; Bregman et al. 1988).

The connections between optical and radio variations have been studied with the very extensive data sets that have been obtained through monitoring efforts. Despite the quantity of available data, firmly establishing optical-radio correlations remains difficult for a variety of reasons. While radio variations in the $\mathrm{cm}$ region are well-resolved (e.g., Aller et al. 1985), the optical variations are undersampled so that outbursts may pass undetected. Furthermore, variations in the optical region do not evolve into easily recognizable radio flares. The amplitude and duration of outbursts in the two wavebands are often quite different (e.g., Valtaoja et al. 1988), and even the variability properties in the two regions are distinct (as measured by a structure function or power spectrum analysis; Bregman and Hugnagel 1989). Despite these problems, it is still possible to establish optical-radio correlations. From about a decade of optical and radio data, Phomphrey (1976) found that only OJ 287 showed a correlation in a sample of about ten objects. Balonek (1982) argued that optical outbursts probably preceded radio variability in objects other than OJ 287, although no statistical treatment was provided. Recently, Bregman and Hufnagel (1989) used more extensive time series (up to 20 years of data) and applied a statistical 
analysis that appears to give robust results. They found that correlated opticalradio variability is common, with the optical variations preceding the radio variations by typically a year. In some cases, such as OJ 287 during certain epochs, the time delay may be 2 months (optical-mm correlations; Valtaoja et al. 1988, 1989), and possibly less (Kikuchi et al. 1988). We conclude that variations in the optical and radio wavebands are causally connected, that the optical variations precede the radio variations by typically months to years, and that considerable processing of the emitting plasma occurs between the optical and the radio regions.

Within the radio domain, variations between the $\mathrm{cm}$ and $\mathrm{mm}$ regions are very well correlated, with outbursts at the higher frequencies preceding those at the lower frequencies by weeks to months at $20-100 \mathrm{GHz}$ frequencies and months to years at 5-15 GHz frequencies (Balonek 1982; Aller et al. 1985; Salonen et al. 1987; Valtaoja et al. 1988; review by Altschuler 1989). In at least one case, $\mathrm{AO} 0235+164$, such correlated variations are successfully followed into the $0.3-1 \mathrm{GHz}$ region (O'Dell et al. 1988), although this is rare.

Depending upon the spectral regions, the reason for the time delays is a combination of opacity effects and the motion of the plasma. Because the synchrotron-self-absorption opacity increases with wavelength, a region must become more dilute before it becomes transparent, at which point it can contribute significantly to the continuum. This would be expected to occur when the emitting region becomes larger and further along the jet axis. However, adiabatic expansion of a plasma component as it moves outward (van der Laan 1966) is inadequate to explain the behavior seen in the radio region and processes involving shocks appear to be required (e.g., Hughes et al. 1989 a, b). The delay time between the optical and radio region probably reflects the distance over which the plasma must travel before it becomes sufficiently dilute that it is transparent to radio photons. If the velocity of the outward moving plasma is approximately the speed of light, then for a Doppler boosting factor $\delta=3$, the distance defined by the time delay is approximately equal to the separation of a superluminal component from the core. This suggests that the origin of superluminal components can be traced to optical activity in the core of the blazar (e.g., Bregman et al. 1986).

When the emitting plasma is extremely dense and compact, opacity effects may even modify the infrared emission (Marscher and Gear 1985), although other mechanisms could cause the optical-infrared time delay. For example, if the nonthermal electron distribution in a parcel of plasma is peaked at some energy, then, as the plasma expands adiabatically, the peak energy (and the resulting peak frequency) decrease with time. When the expansion or motion of the plasma is near the speed of light, the time delay, corrected for relativistic time dilation, is a measure of the relative separation or sizes of the two emitting regions. This picture appears plausible since the sizescale deduced from the time delay is approximately the same as the size of the (larger) lower frequency region.

\subsection{Relativistic beaming}

Relativistic bulk motion of the emitting plasma is inferred from several observations. When interpreting observations in terms of relativistic effects, it is impor- 
tant to distinguish between the angle defined by relativistic bulk motion $\left(\theta \sim \gamma^{-1}\right)$, and the angle into which the flow is directed, which can be considerably greater ( $4 \pi$ for spherical expansion). The most powerful arguments for relativistic motion is provided by the radio emission. Temporal flux variations and VLBI sizes imply brightness temperatures exceeding $10^{12} \mathrm{~K}$, and this high inferred photon density should lead to a strong Compton-scattered X-ray flux, in conflict with observations (e.g., Kellermann and Pauliny-Toth 1981). If relativistic boosting is present, then the high radio brightness temperature and the associated X-ray emission can be appropriately reduced. Another strong argument for relativistic motion is the presence of apparent superluminal motion of radio components, which is generally explained as a relativistic aberration effect due to the bulk motion of the radio plasma (Blandford et al. 1977; Lind and Blandford 1985). Modeling of the superluminal components leads to a Lorentz factor of bulk motion of $\gamma=2-6$ for BL Lacertae objects (e.g., Cohen 1989; Mutel 1989), which is about the same value required to reduce the brightness temperature to a reasonable value. Based upon the distribution of values for $\gamma$ and the frequency with which superluminal motion is detected in samples of apparently randomly oriented radio sources, the opening angle for the flow probably exceeds the angle defined by the bulk motion of $\sim \gamma^{-1}$ (e.g., Blandford 1987).

$\mathrm{X}$-ray variability observations lend further evidence for relativistic motion, where in a few objects, the timescale for X-ray variability is less than the light crossing time for the last stable orbit around a supermassive black hole of mass $\sim 10^{8} M_{\odot}$ (Doxsey et al. 1983; Feigelson et al. 1986; Giommi et al. 1986; Fig. 7). The light crossing time would be suitable lengthened by a Lorentz factor for bulk motion of a few, although limitations of the theory and the observations prevent a more precise determination of $\gamma$.

Recently, several studies have addressed the issue of whether the relativistic bulk motion is a function of wavelength (Stocke et al. 1985; Maraschi et al. 1986; Worrall 1987; Impey and Tapia 1990). Usually, these investigations couch their analysis within a simple jet picture, where the bulk Lorentz factor determines the opening angle of the emitted radiation (Blandford and Konigl 1979; Ghisellini 1985). The orientation of the beamed emission may be estimated by comparing the flux of the beamed component to an isotropic component. The extended or lobe emission is expected to be an isotropic radiator, since both lobes are of similar brightness (see e.g., Bridle and Perley 1984). If the absolute lobe to core luminosity ratio and the Lorentz factor of bulk motion is constant from object to object, then the ratio of the compact to extended flux $(R)$ measures $\theta$, the angle of the jet to our line of sight (e.g., Wills 1989). A correlation is present between $R$ and the percentage of optical polarization $(p)$, which implies that the optical emission is also relativistically boosted (Impey and Tapia 1990). However, the optical polarization does not rise as rapidly as one might expect if the Lorentz factor of bulk motion were equal in the optical and radio region. Relative to the radio emitting plasma, the bulk motion of the optically emitting plasma appears to be smaller.

Progressing toward higher frequencies, the bulk Lorentz factor appears to be lower for the X-ray than for the radio or optical emission. X-ray selected BL Lacertae objects are less polarized in the optical region than radio selected objects and they have weaker core radio emission (lower $R$; Stocke et al. 1985). Since the optical polarization and $R$ are measures of orientation, this result would occur if the opening angle for the X-ray emission is greater than for 


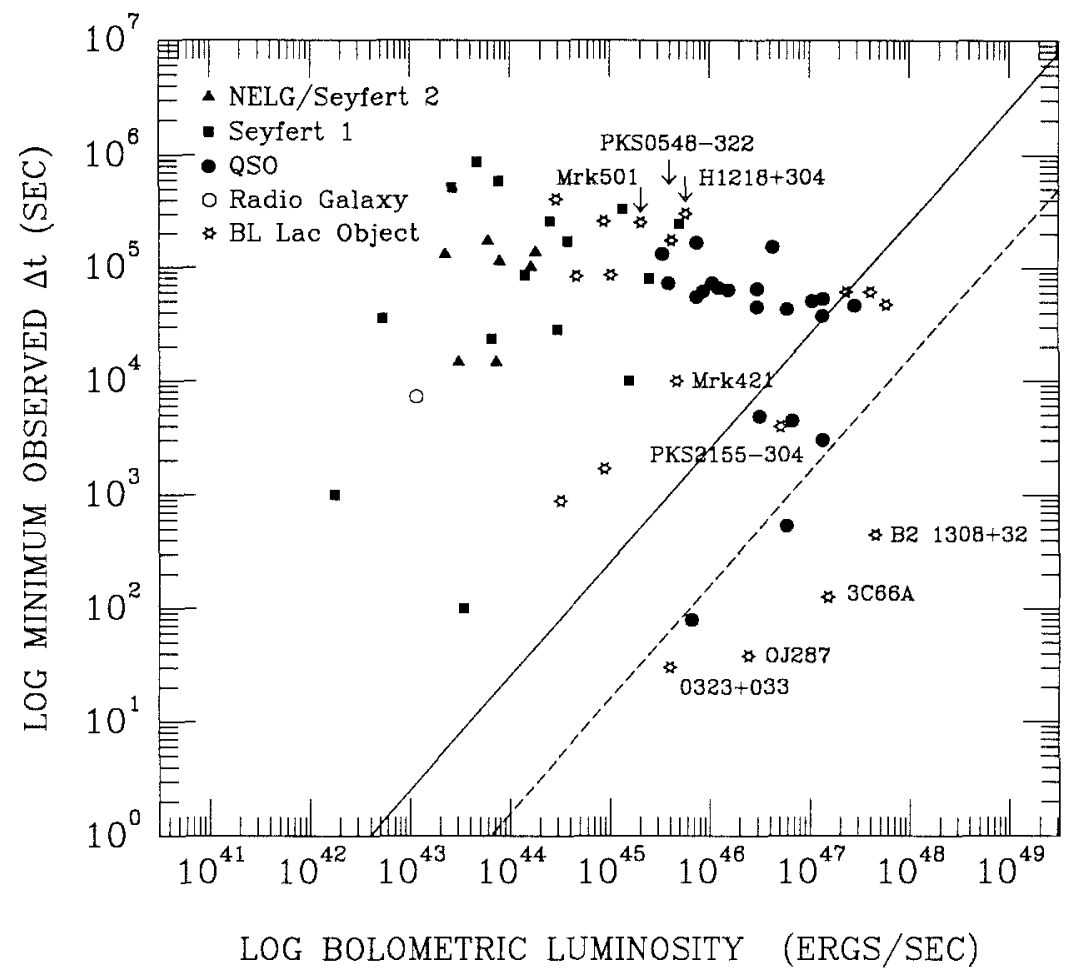

Fig. 7. The minimum timescale of variability, defined as the time in which a $50 \%$ change occurs, is plotted against bolometric luminosity for a broad assortment of AGN (Bassini, Dean, and Sembay 1983; additional objects by Urry, private communication). The left diagonal line is the Eddington luminosity when the size of the Schwarzschild radius is given by the variability time (Elliot and Shapiro 1974). The right diagonal line also gives a maximum expected luminosity in the event that the emission is asymmetric (Abromowitz and Nobili 1982).

the optical or radio emission (equivalently, a lower Lorentz factor for the X-ray emission). If the $\mathrm{X}$-ray emission has a larger opening angle, it should be more widely visible than the optical or X-ray emission, and this expectation is sustained by observation. For a fixed X-ray luminosity, X-ray selected objects have a higher space density than radio selected BL Lacertae objects (Ghisellini et al. 1986; Maraschi et al. 1986), although the samples used are not statistically homogenous. To conclude, the data suggest that the Lorentz factor of bulk motion decreases with increasing frequency from the radio to the $\mathrm{X}$-ray region, although a quantitative measure of this decrease has not yet been obtained.

\subsection{Modeling the synchrotron emitting plasma}

The physical quantities of the emitting plasma can be deduced by interpreting the observations within the framework of a model. Nearly all of the detailed studies have employed the synchrotron-Compton model, in which the radio through ultraviolet emission is produced by nonthermal synchrotron emission 
and the X-ray flux is Compton scattered photons (e.g., Jones et al. 1974). Although the X-ray emission may not be Compton produced in all cases (see below), it can place an upper limit on the Compton scattered contribution.

In the simplest form of the synchrotron-Compton model, a homogeneous volume of plasma is characterized by a single value of the magnetic field $B$, the electron density parameter $k_{\mathrm{e}}$, the size of the emitting region, and the Doppler boosting factor $\delta=\gamma^{-1}(1-\beta \cos \theta)^{-1}$. The observations now force us to consider a much more complex model in which these plasma parameters depend upon position in a jet. Inhomogeneous plasma jet models usually make the assumption that the magnetic field and particle density decrease with distance along the jet according to a power-law distribution: $B \propto r^{-m}, k_{\mathrm{e}} \propto r^{-n}$ (Marscher 1980; Blandford and Konigl 1979; Konigl 1981, 1989; Reynolds 1982; Ghisellini et al. 1985). The permissible values for the exponents $m$ and $n$ can be determined in the radio region from the shape of the spectrum; this yields values of 1 to 2 . Some analyses show that the emission in the $\mathrm{mm}$-sub $\mathrm{mm}$ regions is characterized by $B \sim 1 G, k_{\mathrm{e}}=10^{3}-10^{6}\left(n_{e} \sim 10^{3} \mathrm{~cm}^{-3}\right)$, a size of $10^{17} \mathrm{~cm}$, and electron energies of 10-10 $\mathrm{MeV}$ (e.g., Bregman et al. 1988), although in other analysis of similar objects, $B$ and $k_{\mathrm{e}}$ can vary considerably, generally having lower values (e.g., Urry and Mushotzky 1982; Ghisellini et al. 1985; Hutter and Mufson 1986; Worrall et al. 1986; Mufson et al. 1989). From the adopted radial dependencies of $B$ and $k_{\mathrm{e}}$, the plasma producing optical emission should have a magnetic field and particle density that is $10-10^{3}$ times greater than the above values. Magnetic fields as large as $\sim 10^{3} G$ are implied, which is similar to the energy density expected in an accretion disk (e.g., Begelman 1985), and would be consistent with a picture in which the energetic plasma is created near an accretion disk around a black hole (we temper this speculation with the fact that in BL Lacertae objects, there is no evidence for thermal emission from an accretion disk).

\subsection{The origin of the $X$-ray emission}

The picture of the X-ray emission from blazars has been strongly influenced by a variety of studies of the soft $X$-ray emission and supplemented by investigations of the hard X-ray emission, for which there is relatively less data. Soft $\mathrm{X}$-ray studies conducted with the Einstein IPC show that BL Lacertae objects do not possess the very soft $X$-ray excess seen in ordinary AGN (Wilkes and Elvis 1987; Madejski and Schwartz 1989). The mean soft X-ray spectral slope obtained with the Einstein IPC $(0.1-4 \mathrm{keV})$ is $\left\langle\alpha_{x}\right\rangle=-1.0$, regardless of whether they were radio or X-ray selected objects (Ghisellini et al. 1986; Maraschi et al. 1986; Worrall 1989). The broad range of spectral slopes, from about -0.5 to -2.0 are in contrast to the bright Seyfert galaxies observed with HEAO-1, which showed a "universal" X-ray slope of -0.7 (Mushotzky 1984). Within the blazar group, the spectral slopes of the BL Lacertae objects $\left(\left\langle\alpha_{x}\right\rangle=-1.0\right)$ are generally steeper than for the OVVs and the flat spectrum radio sources $\left(\left\langle\alpha_{x}\right\rangle=-0.6\right)$, indicating that there is no unified blazar class (Worrall 1989a).

Higher energy data obtained with the Einstein MPC $(1.2-10 \mathrm{keV})$ can be combined with the IPC measurements to show that the continua of BL Lacertae objects steepen by about $\Delta \alpha=0.5$ in the energy range $1-3 \mathrm{keV}$ (Madejski and Schwartz 1989). This result is confirmed by observations made with the EXO- 
SAT LE (0.1-2 keV) and ME (2 -6 keV) instruments (Barr et al. 1989; Giommi et al. 1990; Garilli and Maccagni 1990). X-ray spectra in a higher energy region, 2-35 keV, have been obtained with the LAC on the GINGA satellite (Ohashi 1989), and although there are only a handful of objects, the continua can be represented by single power law spectra of mean slope -1.0 (for a sample of BL Lacertae objects and OVVs). Apparently, these observations examine the region above the spectral change.

A feature of potentially great importance is the hard X-ray tail $(2-20 \mathrm{keV}$ range), which has been a topic of some controversy. Following the reports of this feature in data obtained with the HEAO-1 satellite (Urry and Mushotzky 1982), it was searched for in a variety of objects, but never found at a level greater than 3.5 $\sigma$ (Maccagni et al. 1989; Ohashi 1989; Madejski and Schwartz 1989). The duty cycle of this feature must be so low, $<1.5 \%$, that we question whether it is truly present.

The spectral properties of the X-ray emission can be used in conjunction with the radio-ultraviolet continuum to constrain the X-ray production mechanism. For example, multifrequency spectra can be used to examine whether or not the X-ray emission forms a smooth continuation of the lower-frequency synchrotron emission (review by Bregman, Marashi, and Urry 1987; Bregman et al. 1988, 1990; Makino et al. 1987; George et al. 1988; Worrall 1989 a; Makino 1990). When the $X$-ray emission is a smooth continuation of the lower frequency emission, it is likely that the emission mechanism is the synchrotron process. This frequently occurs in BL Lacertae objects that were discovered from their X-ray or optically properties (e.g., Mrk 421, Makino et al. 1987; PKW 2155-304, Treves et al. 1989), which are often the brightest and best studied X-ray blazars. This picture is supported by variability studies, where the X-ray variations are correlated with changes in the optical and ultraviolet region (e.g., 0537-441, Tanzi et al. 1986; PKS 2155-304, Teves et al. 1989). Such efforts show that variability can be more rapid and of greater amplitude at higher frequencies (even in adjacent X-ray bands; Fig. 8), suggesting that the size of the emitting region decreases with increasing frequency.

A particularly interesting class of objects are those where the X-ray emission is unconnected from the lower frequency emission. More precisely, an extension of the IR-UV continuum would underpredict the observed X-ray emission, which usually has a shallower continuum spectral slope. Three examples of such objects are BL Lacertae, 3C 446, and the optically violent variable (OVV) 3C 345 (summarized by Makino 1990), which have $1-35 \mathrm{keV}$ continue with slopes of -0.4 to -0.7 , somewhat shallower than the mean. Here, the X-ray emission arises either from a separate process (i.e., the Compton mechanism), or by the synchrotron process, but in a region spatially distinct from the radioultraviolet emission region.

The various X-ray emission mechanisms have different observational signatures that can be tested by examining X-ray variability in conjunction with variability at other wavebands. In the synchrotron-Compton model for X-ray production, the seed photons that are scattered are expected to originate in the infrared-radio region (e.g., Jones et al. 1974). For power-law electron distributions, the shapes of the scattered and seed photon spectra should match. Also, there should be a close relationship in the variability between the scattered and seed photons. Of the objects for which multiwavelength variability measurements have been obtained, this expectation is satisfied for $0735+178$, BL Lacer- 

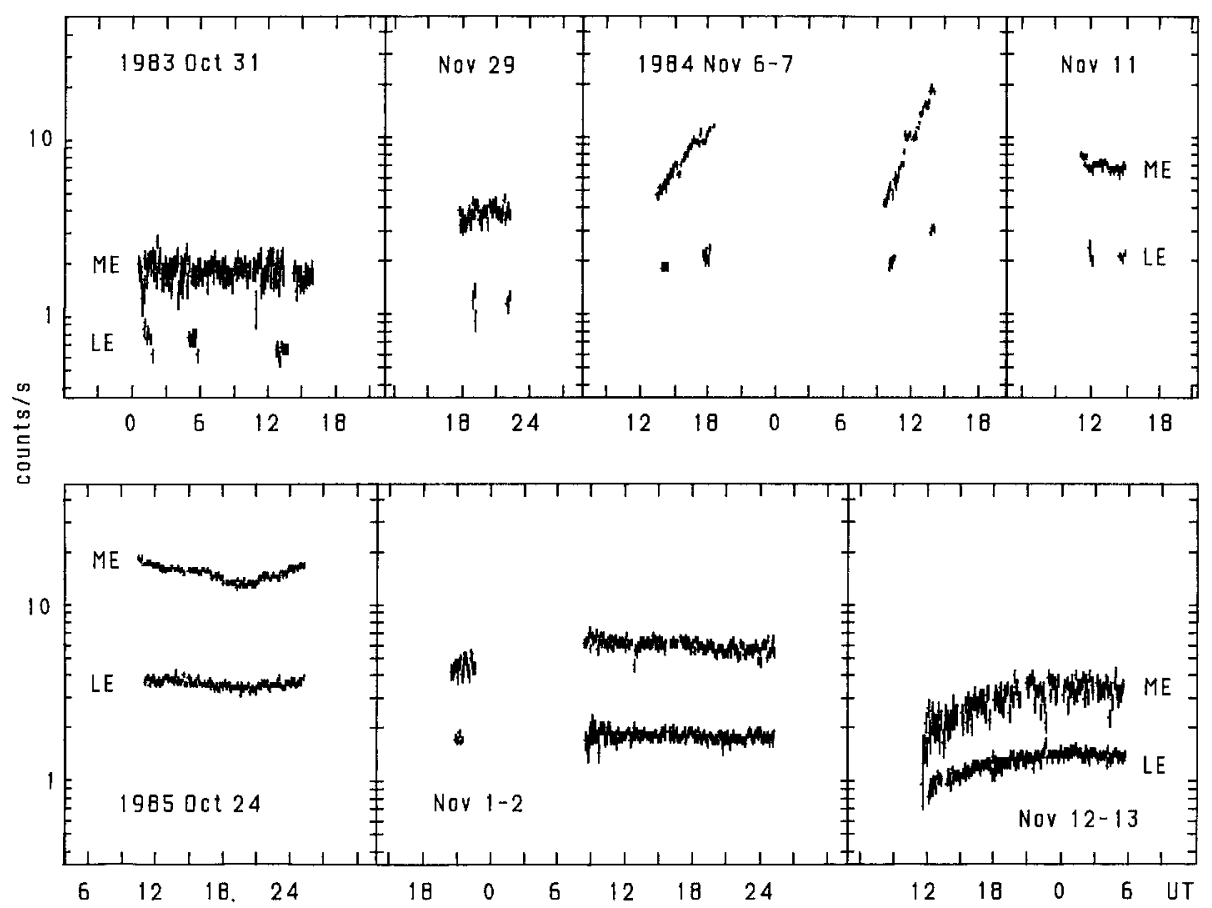

Fig. 8. Variability in the 0.2-0.6 keV EXOSAT LE band and the 1-6 keV EXOSAT ME band for PKS 2155-304 (Treves et al. 1989). Although the variations in both bands are generally well-correlated, larger amplitude variation at higher energies is seen in 1984 Nov 6-7

tae, and 3C 345 (Bregman et al. 1984, 1986, 1990). Furthermore, Kawaii et al. (1989) have shown that the spectral slope of the X-ray and the infrared emission (the likely seed photons) were the same. The correlated variability and the long timescale for X-ray variability in these objects suggest that Compton scattering by relativistic electrons is likely to be active in the production of X-ray photons.

A different type of variability behavior is seen in some objects, suggesting the presence of another X-ray production mechanism in blazars in which the $\mathrm{X}$-ray emission is distinct from the infrared-optical-ultraviolet emission. X-ray variability is correlated with, but varies more rapidly than the optical-ultraviolet emission (3C 446, Bregman et al. 1988; OJ 287, Pollock et al. 1985); it may precede optical variations $(1156+295$, McHardy 1989). Such variability indicates that the $\mathrm{X}$-ray emission region is smaller than anticipated from the synchrotronself-Compton model. In this case, the pair-cascade model may be active, in which the interaction of soft photons (optical-ultraviolet) with energetic particles and photons in a compact region produces the X-ray emission (e.g., Fabian 1988).

\subsection{The parent population}

In the picture where the radio continuum emission from BL Lacertae objects is relativistically boosted into a cone of small solid angle, a large unbeamed 
parent population must exist. Several investigators have suggested that this parent population is the class of FR I radio galaxies (Wardle et al. 1984; Peacock 1987; Browne 1989; Miller 1989; Woltjer 1989; Ulrich 1989; Urry 1990). The underlying galaxies of the FR Is and the BL Lacertae objects are qualitatively similar (giant elliptical galaxies), as are the extended radio fluxes. Within the beaming model, the ratio of the space densities of the parent and beamed populations should provide an estimate for the opening angle to the cone in which beaming occurs. Recently, Woltjer (1989) has estimated that the local space density of BL Lacertae objects is no greater than $80 \mathrm{Gpc}^{-3}$ and that the space density of FR I radio galaxies is about $3000 \mathrm{Gpc}^{-3}$. This implies an opening half angle of $\theta_{0} \approx 13^{\circ}$ (double-sided jet), which either defines the shape of the cone within which flow occurs $\left(\theta_{\text {cone }}=\theta_{0} ; \theta_{\text {cone }}>\gamma_{\text {bulk }}^{-1}\right)$, or the relativistic bulk motion in the case of narrow cones (for $\theta_{\text {cone }}<\gamma_{\text {bulk }}^{-1}, \theta_{\text {cone }} \approx \gamma_{\text {bulk }}^{-1}$ ). It is not yet possible to eliminate one of these models, but if the latter case is valid, we infer a value of $\gamma_{\text {bulk }} \approx 4$ (half angle at half maximum), which is similar to the values $\gamma_{\mathrm{bulk}} \sim 2-6$ that have been independently estimated from the VLBI, multifrequency and variability studies.

Relativistic beaming should also flatten a steep luminosity distribution function of the parent population (Padovani and Urry 1990). The data are consistent with this prediction, although a statistical study will be required to test this expectation quantitatively.

This "unification" of BL Lacertae objects with FR I radio galaxies is parallel to the unification of FR II radio galaxies with radio loud quasars (including OVV quasars) discussed above $(\$ 2.6)$. If Seyfert 1 and 2 type galaxies are also a single object seen at different orientations $(\$ 2.6)$, then there are very few independent phenomena responsible for continuum emission of AGN. All AGN could be composed of combinations of (1) a jet (of either high or low power) of nonthermal material in which relativistic Doppler boosting occurs, and (2) a thermal accretion disk with an obscuring cone or torus, an emission line region, and a surrounding dusty ISM.

\subsection{Model testing}

The plasma jet picture of blazars is well supported and appears to be successful in accommodating a wide range of observations. An important next step would be to obtain a quantitative distribution of the plasma properties $B, k_{\mathrm{e}}, \delta$, and the electron energy distribution as a function of position. Ideally, this information should be obtained through imaging of the emitting region as a function of time and with micro-arcsecond spatial resolution. Until this becomes technically feasible, the best approach to obtaining this information is probably coordinated variability studies in which several outbursts are time resolved from the radio region through the ultraviolet or optical region. Previous variability efforts either were not coordinated or the sampling in a crucial spectral region (e.g., optical) was entirely inadequate. With a carefully designed program, a coordinated effort between the groups currently involved with monitoring blazars should be able to provide the desired observations.

A complementary theoretical effort will be needed to utilize the full assortment of constraints provided by the observations, some of which already exist. In particular, future efforts should attempt to reproduce not only the continuum 
data, but the variability observations and the apparent decrerase in the opening angle with frequency. Efforts to accommodate such information in models have begun (e.g., Ghisellini and Maraschi 1989; Celotti et al. 1989), but it is necessary for these models to become more comprehensive. It is also essential to understand the sensitivity (reliability) of the deduced quantities to the model assumptions.

Another issue is whether shocks merely compress the plasma, as is argued in the radio emitting region (e.g., Marscher and Gear 1985; Hughes et al. 1989a, b), or whether they produce significant numbers of energetic particles. One particle production mechanism is the shock acceleration model (see review by Blandford and Eichler 1987), from which the resulting spectrum has been discussed in the quasar environment (Bell 1978; Blandford and Ostriker 1978; Webb et al. 1984; Bregman 1985; Heavens and Meisenheimer 1987; Fritz 1989). It should be possible to observationally test this model, especially the straightforward predictions of the multicolor variability (Bregman and Boisseau 1989).

One of the most difficult problems to address has been the origin of the high energy continuum radiation. While coordinated variability studies find evidence supporting an inverse Compton origin for the soft X-rays, counterexamples indicate that other mechanisms may be present. An alternative mechanism is the pair cascade model, in which $\gamma$-rays scatter off soft (optical-UV) photons to produce the soft X-ray emission (e.g., Fabian 1988). It should be possible to discriminate between these two models by multifrequency variability studies and by statistical studies of the distribution of X-ray spectral slopes (other models are possible, e.g., Jones and Stein 1987, 1989). Finally, a hard X-ray component is predicted by a model in which the observed velocity of the superluminal components is a consequence of Compton drag against the jet (Melia and Konigl 1989). Although hard X-ray components (tails) were seen in some HEAO-1 observations, the evidence for these features is currently weak $(\$ 3.6)$. A definitive search for this feature, preferably during the emergence of a new superluminal component, would be of great value in testing this model.

Acknowledgements. The author is grateful for a number of illuminating discussion with W. Mathews, R. Edelson, M. Malkan, K. Pounds, A. Bridle, M. Elvis, L. Maraschi, D. Sanders, S. Phinney, D. Richstone, H. Aller, G. MacAlpine, and B. Wills, as well as a thoughtful reading of this work by P. Hughes. In addition, the author is indebted to L. Woltjer for his understanding and advise in the completion of this work.

\section{References}

Abramowicz, M.A., Nobili, L.: 1982, Nature 300, 506

Agrawal, P.C., Singh, K.P., Riegler, G.R.: 1987, Monthly Notices Roy. Astron. Soc. 227, 525

Aitken, D.K., Roche, P.F.: 1985, Monthly Notices Roy. Astron. Soc. 213, 777

Aller, H.D., Adler, M.F., Latimer, G.E., Hodge, P.E. : 1985, Astrophys. J. Suppl. 59, 513

Altschuler, D.R.: 1982, Astron. J. 87, 387

Altschuler, D.R.: 1983, Astron. J. 88, 16

Altschuler, D.R.: 1989, Fund. of Cosmic Physics 14, 37

Antonucci, R.R.J.: 1984, Astrophys. J. 278, 299

Antonucci, R.R.J., Barvainis, R.: 1988, Astrophys. J. Letters 322, L13

Antonucci, R.R.J., Kinney, A.L., Ford, H.C.: 1989, Astrophys. J. 342, 64

Antonucci, R.R.J., Miller, J.S.: 1985, Astrophys. J. 297, 621

Antonucci, R.R.J., Hickson, P., Miller, J.S., Olszewski, E.W.: 1987, Astron. J. 93, 785 
Arnaud, K.A. et al.: 1985, Monthly Notices Roy. Astron. Soc. 217, 105

Axon, D.J., Unger, S.W., Pedlar, A., Meurs, E.J.A., Whittle, D.M., Ward, M.J.: 1989, Nature 341, 631

Babadyhanzants, M.K., Belokon', E.T.: 1984, Astrophysics 21, 461

Babadyhanzants, M.K.: 1986, Astrophysics 23, 639

Ballard, K.R. et al.: 1989, in BL Lac Objects, eds. Maraschi, L., Maccacaro, T., Ulrich, M.-H.: Springer, Berlin Heidelberg New York, p. 181

Ballard, K.R., Mead, A.R.G., Brand, P.W.J.L., Hough, J.H.: 1990, Monthly Notices Roy. Astron. Soc. 243, 640

Balonek, T.J.: 1982, Ph. D. Thesis, University of Massachusetts

Balzano, V.A., Weedman, D.W.: 1981, Astrophys. J. 243, 756

Barbieri, C., Cappellaro, E., Romano, G., Turatto, M., Szuszkiewicz, E.: 1988, Astron. Astrophys. Suppl. 76, 477

Barr, P., Giommi, P., Pollock, A., Tagliaferri, G., Maccagni, D., Garilli, B. 1989, in BL Lac Objects, eds. Maraschi, L., Maccacaro, T., Ulrich, M.-H.: Springer, Berlin Heidelberg New York, p. 290

Barstow, M.A., Stanger, V.J.: 1988, Monthly Notices Roy. Astron. Soc. 230, 207

Barthel, P.D.: 1989, Astrophys. J. 336, 606

Barvainis, R.: 1987, Astrophys. J. 320, 537

Barvainis, R., Alloin, D., Antonucci, R.: 1989, Astrophys. J. Letters 337, L69

Barvainis, R., Antonucci, R.: 1989, Astrophys. J. Suppl. 70, 173

Bassini, L., Dean, A.J., Sembay, S.: 1983, Astron. Astrophys. 125, 52

Bechtold, J., Czerny, B., Elvis, M., Fabbiano, G., Green, R.F.: 1987, Astrophys. J. 314, 699

Begelman, M.C.: 1985, in Astrophysics of Active Galaxies and Quasi-Stellar Objects, ed. Miller, J.S., Mill Valley: University Science Books, p. 411

Begelman, M.C., Blandford, R.D., Rees, M.J.: 1984, Rev. Mod. Phys. 56, 255

Beichman, C.A., Neugebauer, G., Soifer, B.T., Wootten, H.A., Roellig, T., Harvey, P.M.: 1981 a, Nature 293, 711

Beichman, C.A., Provdo, S.H., Neugebauer, G., Soifer, B.T., Matthews, K., Wootten, H.A.: 1981 b, Astrophys. J. 247, 780

Bell, A.R.: 1978, Monthly Notices Roy. Astron. Soc. 182, 147

Bezler, M., Gruber, D.E., Rothschild, R.E.: 1988, Astrophys. J. 334, 995

Biretta, J.A., Moore, R.L., Cohen, M.H.: 1986, Astrophys. J. 308, 93

Blandford, R.D.: 1987, in Superluminal Radio Sources, eds. Zensus, J.A., Pearson, T.J., Cambridge Press, Cambridge, p. 40

Blandford, R.D., Eichler, D.: 1987, Physics Reports, 154, 1

Blandford, R.D., Konigl, A.: 1979, Astrophys. J. 232, 34

Blandford, R.D., McKee, C.F., Rees, M.J.: 1977, Nature 267, 563

Blandford, R.D., Ostriker, J.P.: 1978, Astrophys. J. (letters) 221, L29

Botti, L.C.L., Abraham, Z.: 1987, Rev. Mex. Astron. Astrofis. 14, 97

Branduardi-Raymont, G., Mittaz, J.P.D.: 1988, Adv. Space Res. 8, 61

Bregman, J.N.: 1985, Astrophys. J. 288, 32

Bregman, J.N., Boisseau, J.R.: 1989, Astrophys. J. 247, 118

Bregman, J.N., Hufnagel, B.R.: 1989, in BL Lac Objects, eds. Maraschi, L., Maccacaro, T., Ulrich, M.-H., Springer, Berlin Heidelberg New York, p. 159

Bregman, J.N. et al.: 1981, Nature 293, 714

Bregman, J.N. et al.: 1984, Astrophys. J. 276, 454

Bregman, J.N. et al.: 1986, Astrophys. J. 301, 708

Bregman, J.N. et al.: 1988, Astrophys. J. 331, 746

Bregman, J.N. et al.: 1990, Astrophys. J. 352, 574

Bregman, J.N., Maraschi, L., Urry, C.M.: 1987, in Scientific Accomplishments of the IUE, ed. Kondo, Y., Reidel, Dordrecht, p. 685

Bridle, A.H.: 1987, in Active Galactic Nuclei, eds. Miller, H.R., Wiita, P.J., Springer, Berlin Heidelberg New York, p. 329

Bridle, A.H., Perley, R.A.: 1984, Ann. Rev. Astron. Astrophys. 22, 319

Brindle, C. et al.: 1985, Monthly Notices Roy. Astron. Soc. 214, 619

Brock, D., Joy, M., Lester, D.F., Harvey, P.M., Ellis, H.B.: 1988, Astrophys. J. 329, 208

Brown, L.M.J. et al.: 1989 a, Astrophys. J. 340, 129 
Brown, L.M.J., Robson, E.I., Gear, W.K., Smith, M.G.: 1989 b, Astrophys. J. 340, 162

Browne, I.W.A.: 1989, in BL Lac Objects, eds. Maraschi, L., Maccacaro, T., Ulrich, M.-H., Springer, Berlin Heidelberg New York, p. 401

Brunner, H., Worrall, D.M., Wilkes, B.J., Elvis, M.: 1989, Proceedings of the 23d ESLAB Symposium, (ESA SP. 296), p. 905

Burstein, D., Heiles, C.: 1982, Astron. J. 87, 1165

Carini, M.T.: 1990, Ph. D. Thesis, Georgia State University

Carleton, N.P., Elvis, M., Fabbiano, G., Willner, S.P., Lawrence, A., Ward, M.: 1987, Astrophys. J. 18, 595

Cawthorne, T.V., Wardle, J.F.C.: 1988, Astrophys. J. 332, 696

Celotti, A., Maraschi, L., Treves, A.: 1989, in BL Lac Objects, eds. Maraschi, L., Maccacaro, T., Ulrich, M.-H., Springer, Berlin Heidelberg New York, p. 332

Cheng, F.H., Shields, G.A., Lin, D.N.C., Pringle, J.: 1988, Astrophys. J. 328, 223

Chini, R., Biermann, P.L., Kreysa, E., Kuhr, H., Mezger, P.G., Schmidt, J., Witzel, A., Zensus, J.A.: 1987, Astron. Astrophys. 181, 237

Chini, R., Krerysa, E., Salter, C.J.: 1987, Astron. Astrophys. 182, L63

Chini, R., Steppe, H., Kreysa, E., Krichbaum, T., Quirrenbach, A., Schalinski, C., Witzel, A.: 1988, Astron. Astrophys. 192, L1

Chini, R., Kreysa, E., Biermann, P.L.: 1989, Astron. Astrophys. 219, 87

Clavel et al.: 1989, Astrophys. J. 337, 236

Clegg, P.E. et al.: 1983, Astrophys. J. 273, 58

Clement, R., Sembay, S., Hanson, C.G., Coe, M.J.: 1988, Monthly Notices Roy, Astron. Soc. 230, 117

Cohen, M.H.: 1989, in BL Lac Objects, eds. Maraschi, L., Maccacaro, T., Ulrich, M.-H., Springer, Berlin Heidelberg New York, p. 13

Coleman, H.H., Shields, G.A. : 1990, Astrophys. J. (in press)

Corso, G.J. et al.: 1988, Pub. Astron. Soc. Pacific 100, 70

Courvoisier, T.J.-L. et al.: 1987, Astron. Astrophys. 176, 197

Courvoisier, T.J.-L. et al.: 1988, Nature 335, 330

Courvoisier, T.J.-L. et al.: 1990, Astron. Astrophys. (in press)

Czerny, B., Elvis, M.: 1987, Astrophys. J. 321, 305

Done, C., Ward, M.J., Fabian, A.C., Kunieda, H., Tsuruta, S., Lawrence, A., Smith, M.G., Wamsteker, W.: 1990, Monthly Notices Roy. Astron. Soc. 243, 713

Doxsey, R. et al.: 1983, Astrophys. J. Letters 264, L43

Draine, B.T., Anderson, N.: 1985, Astrophys. J. 292, 494

Eales, S.A., Arnaud, K.A.: 1988, Astrophys. J. 324, 193

Edelson, R.A., Gear, W.K.P., Malkan, M.A., Robson, E.I.: 1988, Nature 336, 749

Edelson, R.A., Malkan, M.A.: 1986, Astrophys. J. 308, 59

Edelson, R.A., Malkan, M.A.: 1987, Astrophys. J. 323, 516

Edelson, R.A., Malkan, M.A., Rieke, G.H.: 1987, Astrophys. J. 321, 233

Elias, J.H. et al.: 1978, Astrophys. J. 220, 25

Elliot, J.L., Shaprio, S.L.: 1974, Astrophys. J. Letters 192, L3

Elvis, M.: 1989, Comments in Astrophysics 14, 177

Elvis, M., Green, R.F., Bechtold, J., Schmidt, M., Neugebauer, G., Soifer, B.T., Matthews, K., Fabbiano, G.: 1986, Astrophys. J. 310, 291

Elvis, M., Wilkes, B.J., McDowell, J.C.: 1990, in Extreme Ultraviolet Astronomy, ed. Malina, R.F., Bowyer, S, Pergamon, New York (in press)

Elvis, M., Wilkes, B.J., Tananbaum, H.: 1985, Astrophys. J. 292, 357

Engargiola, G., Harper, D.A., Elvis, M., Willner, S.P.: 1988, Astrophys. J. Letters 322, L19

Epstein, E.E., Fogarty, W.G., Mottmann, J., Schneider, E.: 1982, Astron. J. 87, 449

Fabian, A.C.: 1988, in Supermassive Black Holes, ed. Kafatos, M., Cambridge Univ. Press, Cambridge, p. 234

Fiedler, R.L. et al.: 1987, Astrophys. J. Suppl., 65, 319

Feigelson, E.D. et al.: 1986, Astrophys. J. 302, 337

Flett, A.M., Henderson, C.: 1981, Monthly Notices Roy. Astron. Soc. 194, 961

Fritz, K.D.: 1989, Astron. Astrophys. 214, 14

Gabuzda, D.C., Cawthorne, T.V., Roberts, D.H., Wardle, J.F.C.: 1989, Astrophys. J. 347, 701

Gabuzda, D.C., Wardle, J.F.C., Roberts, D.H.: 1989, Astrophys. J. Letters 336, 59 
Garilli, B., Maccagni, D.: 1990, Astron. Astrophys. 229, 88

Garrinton, S.T., Leahy, J.P., Conway, R.G., Laing, R.A.: 1988, Nature 331, 147

Gear, W.K. et al.: 1985, Astrophys. J. 291, 511

Gear, W.K., Brown, L.M.J., Robson, E.I., Ade, P.A.R., Griffin, M.J., Smith, M.G., Nolt, I.G., Radostitz, J.V., Veeder, G., Lebofsky, L.: 1986, Astrophys. J. 304, 295

George, I.M., Warwick, R.S., Bromage, G.E.: 1988, Monthly Notices Roy. Astron. Soc. 232, 793

Ghisellini, G., Maraschi, L., Tanzi, E., Treves, A.: 1986, Astrophys. J. 310, 317

Ghisellini, G., Maraschi, L., Tanzi, E., Treves, A.: 1985, Astron. Astrophys. 146, 204

Ghisellini, G., Maraschi, L.: 1989, Astrophys. J. 340, 181

Giommi, P. et al.: 1986, Astrophys. J. 303, 596

Giommi, P. et al.: 1987, Astrophys. J. 322, 662

Giommi, P., Barr, P., Garilli, B., Maccagni, D., Pollock, A.M.T.: 1990, Astrophys. J. (in press)

Glassgold, A.E. et al.: 1983, Astrophys. J. 274, 101

Goodrich, R.W.: 1989, Astrophys. J. 340, 190

Goodrich, R.W., Miller, J.S.: 1988, Astrophys. J. 331, 332

Griffiths, R.E., Wilson, A.S., Ward, M.J., Tapia, S., Ulvestad, J.: 1989, Monthly Notices Roy. Astron. Soc. 240, 33

Hagen-Thorn, V.A., Denisenko, N.S., Maksimova, T.M., Marchenko, S.G., Mikolajchuk, O.V.: 1987, Tr. Astron. Obs., Tom. Leningrad 41, 96

Halpern, J.: 1982, Ph. D. Thesis, Harvard University

Haniff, C.A., Wilson, A.S., Ward, M.J.: 1988, Astrophys. J. 334, 104

Heavens, A.F., Meisenheimer, K.: 1987, Monthly Notices Roy. Astron. Soc. 225, 335

Heeschen, D.S., Krichbaum, T., Schalinski, C.J., Witzel, A.: 1987, Astron. J. 94, 1493

Hildebrand, R.H., Whitcomb, S.E., Winston, R., Stiening, R.F., Harper, D.A., Moseley, S.H.: 1977, Astrophys. J. 216, 698

Hughes, P.A., Aller, H.D., Aller, M.F.: 1989 a, Astrophys. J. 341, 54

Hughes, P.A., Aller, H.D., Aller, M.F.: 1989 b, Astrophys. J. 341, 68

Hutchings, J.B., Janson, T., Neff, S.G.: 1989, Astrophys. J. 342, 660

Hutter, D.J., Mufson, S.L.: 1986, Astrophys. J. 301, 50

Hyland, H.R., Allen, D.A.: 1982, Monthly Notices Roy. Astron. Soc. 199, 943

Impey, C.D.: 1987 in Superluminal Radio Sources, eds. Zensus, J.A., Peason, T.J. (CUP, Cambridge), p. 233

Impey, C.D., Neugebauer, G.: 1988, Astron. J. 95, 307

Impey, C.D., Tapia, S.: 1990, Astrophys. J. (in press)

Johnston, K.J. et al.: 1984, Astrophys. J. Letters 277, L31

Jones, B., Worrall, D.M., Rodriguez-Espinoza, J.M., Stein, W.A., Gillett, F.C.: 1984, Pub. Astron. Soc. Pacific 96, 692

Jones, T.W., O’Dell, S.L., Stein, W.A.: 1974, Astrophys. J. 188, 353

Jones, T.W., Stein, W.A.: 1987, Astrophys. J. Letters 320, L1

Jones, T.W., Stein, W.A.: 1990, Astrophys. J. 349, 443

Kaastra, J.S., Barr, P.: 1989, Astron. Astrophys. 226, 59

Kawaii, N. et al.: 1990, in the 23rd ESLAB Symposium, Two Topics in X-Ray Astronomy (in press)

Kellermann, K.I., Pauliny-Toth, I.I.K.: 1981, Ann. Rev. Astron. Astrophys. 19, 373

Kellermann, K.I., Sramek, R., Shaffer, D., Green, R.F., Schmidt, M.: 1989, Astron. J. 98, 1195

Kidger, M.K.: 1988, Pub. Astron. Soc. Pacific 100, 1248

Kikuchi, S.: 1989, in BL Lac Objects, eds. Maraschi, L., Maccacaro, T., Ulrich, M.-H., Springer, Berlin, p. 131

Kikuchi, S., Inoue, M., Mikami, Y., Tabara, H., Kato, T.: 1988, Astron. Astrophys. 190, L8

Kolykhalov, P.I., Sunyaev, R.A.: 1984, Adv. Space Res. 3, 249

Konigl, A.: 1981, Astrophys. J. 243, 700

Konigl, A.: 1989, in BL Lac Objects, eds. Maraschi, L., Maccacaro, T., Ulrich, M.-H., Springer, Berlin, p. 321

Krolik, J.H., Begelman, M.C.: 1986, Astrophys. J. Letters 308, L55

Krolik, J.H., Begelman, M.C.: 1988, Astrophys. J. 329, 702

Krugel, E., Chini, R., Kreysa, E., Sherwood, W.A.: 1988, Astron. Astrophys. 193, L16

Krugel, E., Kreysa, E., Gemund, H.-P.: 1989, Astron. Astrophys. 216, L5 
Kruper, J.S., Urry, C.M., Canizares, C.R.: 1990, Astrophys. J. Suppl. (in press)

Kulshretha, A., Deshpande, M.R., Hoshi, U.C.: 1987, Astron. Astrophys. Suppl. 71, 565

Laing, R.A.: 1988, Nature 331, 149

Landau, R. et al.: 1986, Astrophys. J. 308, 78

Laor, A., Netzer, H.: 1989, Monthly Notices Roy. Astron. Soc. 238, 897

Laor, A., Netzer, H., Piran, T.: 1990, Monthly Notices Roy. Astron. Soc. 242, 560

Lawrence, A.: 1987, Pub. Astron. Soc. Pacific 99, 309

Lawrence, A., Pounds, K.A., Watson, M.G., Elvis, M.S.: 1987, Nature 325, 692

Lawrence, A.: 1988, in Comets to Cosmology, Ed. Lawrence, A., Springer, Berlin, p. 217

Lawrence, A., Rowan-Robinson, M., Leech, K., Jones, D.H.P., Wall, J.V.: 1989, Monthly Notices Roy. Astron. Soc. 240, 329-348

Lebofsky, M.J., Rieke, G.H.: 1980, Nature 284, 410

Lind, K.R., Blandford, R.D.: 1985, Astrophys. J. 295, 358

Lloyd, C.: 1984, Monthly Notices Roy. Astron. Soc. 209, 697

Lorenzetti, D., Massaro, E., Perola, G.C., Spinoglio, L.: 1987, Mem. Soc. Astron. Ital. 58, 279

MacAlpine, G.M.: 1985, in Astrophysics of Active Galaxies and Quasi-Stellar Objects, ed. Miller, J.S., University Science Books, Mill Valley, p. 259

MacAlpine, G.M., Davidson, K., Gull, T.R., Wu, C.-C.: 1985, Astrophys. J. 294, 147

Maccacaro, T., Gioia, I.M., Wolter, A., Zamorani, G., Stocke, J.T.: 1988, Astrophys. J. 326, 680

Madejski, G.M., Schwartz, D.A.: 1989, in BL Lac Objects, eds. Maraschi, L., Maccacaro, T., Ulrich, M.-H. Springer, Berlin, p. 267

Makino, F.: 1990, in the 23rd ESLAB Symposium: Two Topics in X-Ray Astronomy (in press)

Makino, F. et al.: 1987, Astrophys. J. 313, 662

Makino, F. et al.: 1989, Astrophys. J. Letters 347, L9

Malkan, M.A.: 1983, Astrophys. J. 268, 582

Malkan, M.A.: 1988, Adv. Space Res. 8, 49

Malkan, M.: 1989, in Dynamics of Astrophysical Disks, ed. Sellwood, J., Cambridge Univ. Press, Cambridge (in press)

Malkan, M.A., Sargent, W.L.W.: 1982, Astrophys. J. 254, 22

Maraschi, L.: 1988, Adv. Sp. Res., Vol. 8, No. 2-3, 85-94

Maraschi, L., Ghisellini, G., Tanzi, E., Treves, A.: 1986, Astrophys. J. 310, 325

Maraschi, L., Molendi, S.: 1990 (preprint)

Marscher, A.P.: 1980, Astron. J. 235, 386

Marscher, A.P., Gear, W.K.: 1985, Astrophys. J. 298, 114

Martin, P.G., Thompson, I.B., Maza, J., Angel, J.R.P.: 1983, Astrophys. J. 266, 470

McAlary, C.W., Rieke, G.H.: 1988, Astrophys. J. 333, 1

McDowell, J.C., Elvis, M., Wilkes, B.J., Willner, S.P., Oey, M.S., Polomski, E., Bechtold, J., Green, R.F.: 1989, Astrophys. J. 345 Letters, L13

McHardy, I.: 1989, in BL Lac Objects, eds. Maraschi, L., Maccacaro, T., Ulrich, M.-H., Springer, Berlin, p. 189

McHardy, I., Czerny, B.: 1987, Nature 325, 696

Mead, A.R.G., Brand, P.W.J.L., Hough, J.H., Bailey, J.A.: 1988, Monthly Notices Roy. Astron. Soc. 233, 503

Mead, A.R.G., Ballard, K.R., Brand, P.W.J.L., Hough, J.H., Brindle, C., Bailey, J.A.: 1990, Astron. Astrophys. Suppl. 83, 183

Melia, F., Konigl, A.: 1989, Astrophys. J. 340, 162

Meurs, E.J.A., Wilson, A.S.: 1984, Astron. Astrophys. 136, 206

Miley, G.K.: 1980, Ann. Rev. Astron. Astrophys. 18, 165

Miley, G.K., Neugebauer, G., Clegg, P.E., Harris, S., Rowan-Robinson, M., Soifer, B.T., Young, E.: 1984, Astrophys. J. Letters 278, L79

Miley, G.K., Neugebauer, G., Soifer, B.T.: 1985, Astrophys. J. Letters 293, L11

Miller, H.R., Carini, M.T.: 1989, in BL Lac Objects, eds. Maraschi, L., Maccacaro, T., Ulrich, M.-H., Springer, Berlin, p. 143

Miller, J.S.: 1989, in BL Lac Objects, eds. Maraschi, L., Maccacaro, T., U1rich, M.-H., Springer, Berlin, p. 395

Miller, J.S., Antonucci, R.R.J.: 1983, Astrophys. J. Letters 271, L7 
Mitra, A.: 1990, Astrophys. J. 348, 221

Moles, M., Garcia-Pelazo, J.M., Masegosa, J., Garrido, R.: 1986, Astron. J. 92, 1030

Morini, M. et al.: 1986, Astrophys. J. Letters 306, L71

Moore, R.L. et al.: 1982, Astrophys. J. 260, 415

Moore, R.L., Schmidt, G.D., West, S.C.: 1987, Astrophys. J. 314, 176

Moore, R.L., Stockman, H.S.: 1981, Astrophys. J. 243, 60

Moore, R.L., Stockman, H.S.: 1984, Astrophys. J. 279, 465

Mufson, S.L., Hutter, D.J., Kondo, Y.: 1989, in BL Lac Objects, eds. Maraschi, L., Maccacaro, T., Ulrich, M.-H., Springer, Berlin, p. 341

Mushotzky, R.F.: 1982, Astrophys. J. 256, 92

Mushotzky, R.F.: 1984, Adv. Space Res. 3, 157

Mushotzky, R.F.: 1987 in Active Galactic Nuclei, eds. Miller, H.R., Wiita, P.J., Springer, Berlin, p. 239

Mutel, R.L., Phillips, R.B., Su, B., Bucciferro, R.R.: 1990, Astrophys. J. 352, 81

Nandra et al, : 1989, Monthly Notices Roy. Astron. Soc. 236, 39P

Netzer, H.: 1985, Monthly Notices Roy. Astron. Soc. 216, 63

Netzer, H.: 1987, Monthly Notices Roy. Astron. Soc. 225, 55

Neugebauer, G., Becklin, E.E., Oke, J.B., Searle, L.: 1976, Astrophys. J. 205, 29

Neugebauer, G., Oke, J.B., Becklin, E.E., Matthews, K.: 1979, Astrophys. J. 230, 79

Neugebauer, G. et al.: 1984, Astrophys. J. Letters 278, L83

Neugebauer, G., Soifer, B.T., Matthews, K., Elias, J.H.: 1989, Astron. J. 97, 957

Norris, R.P., Allen, D.A., Roche, P.F.: 1988, Monthly Notices Roy. Astron. Soc. 234, 773-782

Norris, R.P., Kesteven, M.J., Allen, D.A., Troupe, E.: 1988, Monthly Notices Roy. Astron. Soc. 234, 51P

O'Brien, P.T., Gondhalekar, P.M., Wilson, R.: 1988a, Monthly Notices Roy. Astron. Soc. 233, 801

O'Brien, P.T., Gondhalekar, P.M., Wilson, R.: 1988 b, Monthly Notices Roy. Astron. Soc. 233, 845

O'Dell, S.L. et al.: 1988 b, Astrophys. J. 326, 668

Ohashi, T.: 1989, in BL Lac Objects, eds. Maraschi, L., Maccacaro, T., Ulrich, M.-H., Springer, Berlin, p. 296

Ohashi, T., Makishima, K., Inoue, H., Koyama, K., Makino, F., Turner, M., and Warwick, R.: 1989, Pub. Astron. Soc. Japan 41, 709

Orr, M.J.L., Browne, I.W.A.: 1982, Monthly Notices Roy. Astron. Soc. 200, 1067

Osterbrock, D.E., Mathews, W.G.: 1986, Ann. Rev. Astron. Astrophys. 24, 171

Owen, F.N.: 1986, in IAU Symposium 119, Quasars, ed. Swarup, G., Kapahi, V.K., Reidel, Dordrecht, p. 173

Pacholczyck, A.G., Wisniewski, W.Z.: 1967, Astrophys. J. 147, 394

Padovani, P., Urry, C.M.: 1990, Astrophys. J. (in press)

Peacock, J.A.: 1987, in Superluminal Radio Sources, eds. Zensus, J.A., Pearson, T.J., Cambridge University Press, Cambridge, p. 12

Penston, M.V., Penston, M.J., Selmes, R.A., Becklin, E.E., Neugebauer, G.: 1974, Monthly Notices Roy. Astron. Soc. 169, 357

Petre, R., Mushotzky, R., Krolik, J., Holt, S.S.: 1984, Astrophys. J. 280, 499

Perez, E., Penston, M.V., Moles, M.: 1989, Monthly Notices Roy. Astron. Soc. 239, 55

Phomphrey, R.B.: 1976, Astron. J. 81, 489

Pica, A.J., Smith, A.G., Pollock, J.T.: 1980, Astrophys. J. 236, 84

Pica, A.J. et al.: 1988, Astrophys. J. 96, 1215

Pogge, R.W.: 1988, Astrophys. J. 328, 519

Pollock, A.M.T., Brand, P.W.J.L., Bregman, J.N., Robson, E.I.: 1985, Sp. Sci. Rev. 40, 607

Pounds, K.: 1985, in Proc. Japan/US Symposium on Galactic and Extragalactic X-Ray Sources, eds. Tanaka, Y., Lewin, W., ISAS, Tokyo, p. 261

Pounds, K.A.: 1989, in Proc. of the $23^{d}$ ESLAB Symposium on X-Ray Astronomy ESA SP-296, p. 753

Pounds, K.A., McHardy, I.M.: 1988, in Physics of Neutron Stars and Black Holes, ed. Tanaka, Y., Univ. Acad. Press, Tokyo, p. 285

Pounds, K.A., Stanger, V.J., Turner, T.J., King, A.R., Czerny, B.: 1986, Monthly Notices Roy. Astron. Soc. 224, 443 
Pounds, K.A., Turner, T.J., Warwick, R.S.: 1986, Monthly Notices Roy. Astron. Soc. 221, $7 \mathrm{P}$

Prestage, R.M., Peacock, J.A.: 1988, Monthly Notices Roy. Astron. Soc. 230, 131

Pustil'nik, S.A., Aliakberov, K.D.: 1987, Astrofiz. Issled. Izv. Spets. Astrofiz. Obs. 25, 68

Rees, M.J.: 1984, Ann. Rev. Astron. Astrophys. 22, 471

Rees, M.J., Silk, J.I., Werner, M.W., Wickramsinghe, M.C.: 1969, Nature 223, 788

Reichert, G., Mushotzky, R., Petre, R., Holt, S.S.: 1985, Astrophys. J. 280, 499

Reynolds, S.P.: 1982 , Astrophys. J. 13

Rieke, G.H.: 1978, Astrophys. J. 226, 550

Rieke, G.H.: 1985, in Astrophysics of Active Galaxies and Quasi-Stellar Objects, ed. Miller, J.S., University Science Books, Mill Valley, p. 236

Rieke, G.H.: 1988, Astrophys. J. Letters 331, L5

Rieke, G.H., Cutri, R.M., Black, J.H., Kailey, W.F., McAlary, C.W., Lebofsky, M.J., Elston, R.: 1985, Astrophys. J. 290, 116

Rieke, G.H., Grasdalen, T.L., Kinman, T.D., Hintzen, P., Wills, B.J., Wills, D.: 1976, Nature 260, 754

Rieke, G.H., Lebofsky, M.J., Kinman, T.D.: 1979, Astrophys. J. Letters 232, L151

Rieke, G.H., Lebofsky, M.J., Thompson, R.I., Low, F.J., Tokunaga, A.T.: 1980, Astrophys. $J .238,24$

Rieke, G.H., Lebofsky, M.J., Wisniewski, W.Z.: 1982, Astrophys. J. 263, 73

Roberts, D.H., Gabuzda, D.C., Wardle, J.F.C.: 1987, Astrophys. J. 323, 536

Robson, E.I., Gear, W.K., Clegg, P.E., Ade, P.A.R., Smith, M.G., Griffin, M.J., Nolt, I.G., Radostitz, J.V., Howard, R.J.: 1983, Nature 305, 194

Robson, E.I., Gear, W.K., Brown, L.M.J., Courvoisier, T.J.-L., Smith, M.G., Griffin, M.J., Blecha, A.: 1986, Nature 323, 134

Roche, P.F., Aitken, D.K., Phillips, M.M., Whitmore, B.: 1984, Monthly Notices Roy. Astron. Soc. 207, 35

Rothschild, R.E., Mushotzky, R.F., Baity, W.A., Gruber, D.E., Matteson, J.L., Primini, F.A.: 1983, Astrophys. J. 269, 423

Rusk, R., Seaquist, E.R.: 1985, Astron. J. 90, 30

Salonen, E. et al.: 1987, Astron. Astrophys. Suppl. 70, 409

Sanders, D.B., Phinney, E.S., Neugebauer, G., Soifer, B.T., Matthews, K.: 1989, Astrophys. J. 347, 29

Sanders, D.B., Soifer, B.T., Elias, J.H., Madore, B.F., Matthews, K., Neugebauer, G., Scoville, N.Z.: 1988 a, Astrophys. J. 325, 74

Sanders, D.B., Soifer, B.T., Elias, J.H., Neugebauer, G., Matthews, K.: 1988b, Astrophys. J. 328, L35

Scheuer, P.A.G., Readhead, A.C.S.: 1797, Nature 277, 182

Schwartz, D.A., Madejski, G.M.: 1987, in Variability of Galactic and Extragalactic X-Ray Sources, ed. Treves A., Associazione per l'Avazamento dell'Astronomia, Milano, p. 65

Shakura, N.I., Sunyaev, R.A.: 1973, Astron. Astrophys. 24, 337

Shields, G.A.: 1978, Nature 272, 706

Shields, G.A., Filippenko, A.V.: 1988, Astrophys. J. Letters 332, L55

Sillanpaa, A., Haarala, S., Korhonen, T.: 1988, Astron. Astrophys. Suppl. 72, 347

Simons, D.A., Tokunaga, A.T., Rudy, R.J., Stein, W.A.: 1988, Astron. J. 96, 481

Singh, K.P., Garmire, G.P., Nousek, J.: 1985, Astrophys. J. 297, 633

Sitko, M.L., Schmidt, G.D., Stein, W.A.: 1985, Astrophys. J. Suppl. 59, 323

Smith, C.H., Aitken, D.K., Roche, P.F.: 1989, Monthly Notices Roy. Astron. Soc. 241, 425

Smith, E.P., Heckman, T.M.: 1990, Astrophys. J. 348, 38

Smith, P.S., Balonek, T.J., Heckert, P.A., Elston, R.: 1986, Astrophys. J. 305, 484

Smith, P.S., Balonek, T.J., Heckert, P.A., Elston, R.: 1987, Astrophys. J. Suppl. 64, 459

Smith, P.S., Elston, R., Berriman, G., Allen, R.G., Balonek, T.J.: 1988, Astrophys. J. Letters 326, L39

Soifer, B.T. et al.: 1984, Astrophys. J. 283, L1

Soifer, B.T., Houck, J.R., Neugebauer, G.: 1987, Ann. Rev. Astron. Astrophys. 25, 187

Solomos, N., Whitehead, M.J., Meaburn, J., Gondis, C.D., Christopoulou, P.E.: 1990, Astron. Astrophys. 229, 80

Steidel, C.C., Sargent, W.L.W.: 1987, Astrophys. J. 313, 171 
Stocke, J.T., Liebert, J., Schmidt, G., Gioia, I., Maccacaro, T., Schild, R., Maccagni, D., Arp, H.: 1985, Astrophys. J. 298, 619

Stocke, J.T., Morris, S.L., Gioia, I., Maccacaro, T., Schild, R.E., Wolter, A.: 1990, Astrophys. $J .348,141$

Stockman, H.S., Moore, R.L., Angel, J.R.P.: 1984, Astrophys. J, 279, 485

Sun, W.-H., Malkan, M.A.: 1987, in Supermassive Black Holes, ed. Kafatos, M., Cambridge Univ. Press, Cambridge, p. 273

Sun, W.-H., Malkan, M.A.: 1989, Astrophys. J. 346, 68

Tagliaferri, G., Stella, L., Maraschi, L., Treves, A., Morini, M.: 1989, in BL Lac Objects, eds. Maraschi, L., Maccacaro, T., Ulrich, M.-H., Springer, Berlin Heidelberg New York, p. 314

Tanzi, E.G. et al.: 1986, Astrophys. J. Letters 311, L13

Telesco, C.M.: 1988, Ann. Rev. Astron. Astrophys. 26, 343

Telesco, C.M., Harper, D.A.: 1980, Astrophys. J. 235, 392

Terasranta, H. et al.: 1987, Astron. Astrophys. Suppl. 71, 125

Thompson, I.B., Martin, P.G.: 1988, Astrophys. J. 330, 121

Thorne, K.S., Page, D.N.: 1974, Astrophys. J. 191, 507

Treves, A., Chiapetti, L., Fabian, A., Falomo, R., Maccagni, D., Maraschi, L., Morini, M., Tanzi, E.G., Tagliaferri, G.: 1989, Astrophys. J. 341, 733

Turner, T.J., Pounds, K.A.: 1988, Monthly Notices Roy. Astron. Soc. 232, 463

Turner, T.J., Pounds, K.A.: 1989, Monthly Notices Roy, Astron. Soc. 240, 833

U1rich, M.-H.: 1989, in BL Lac Objects, eds. Maraschi, L., Maccacaro, T., Ulrich, M.-H., Springer, Berlin Heidelberg New York, p. 435

Ulrich, M.-H., Courvoisier, T.J.-L., Wamsteker, W.: 1988, Astron. Astrophys. 204, 21

Ulvestad, J.S., Wilson, A.S.: 1984, Astrophys. J. 285, 439

Unger, S.W., Pedlar, A., Axon, D.J., Whittle, M., Meurs, E.J.A., Ward, M.J.: 1987, Monthly Notices Roy. Astron. Soc. 228, 671

Urry, C.M., Kondo, Y., Hackney, K.R.H., Hackney, R.H.: 1988, Astrophys. J. 330, 791

Urry, C.M., Mushotzky, R.F.: 1982, Astrophys. J. 253, 38

Urry, C.M., Arnaud, K., Edelson, R.A., Kruper, J.S., Mushotzky, R.F.: 1990, in the proceedings of the 23rd ESLAB Symposium: X-Ray Astronomy (in press)

Valtaoja, E., Haarala, S., Lehto, H., Valtaoja, L., Valtonen, M., Moiseev, I.G., Nesterov, N.S., Salonen, E., Terasranta, H., Urpo, S., Tiuri, M.: 1988, Astron. Astrophys. 203, 1

Valtaoja, L., Sillanpaa, A., Valtaoja, E., Efimov, Y., Shakhavskoy, N.: 1989, in BL Lac Objects, eds. Maraschi, L., Maccacaro, T., Ulrich, M.-H., Springer, Berlin Heidelberg New York, p. 127

van der Laan, H.: 1966, Nature 211, 1131

Wade, R.A.: 1984, Monthly Notices Roy. Astron. Soc. 208, 381

Walker, R.C., Benson, J.M., Unwin, S.C.: 1987, in Superluminal Radio Sources, eds. Zensus, J.A., Pearson, T.J., Cambridge University Press, Cambridge, p. 48

Wandel, A., Petrosian, V.: 1988, Astrophys. J. Letters 329, L11

Wardle, J.F.C., Moore, R.L., Angel, J.R.P.: 1984, Astrophys. J. 279, 93

Warwick, R.S., Koyama, K., Inoue, H., Takano, S., Awaki, H., Hoshi, R.: 1989, Pub. Astron. Soc. Japan 41, 739

Webb, G.M., Drury, L.O'C., Biermann, P.: 1984, Astron. Astrophys. 137, 185

Webb, J.R.: 1990, Astron. J.99, 49

Weedman, D.W.: 1983, Astrophys. J. 266, 479

Weedman, D.W.: 1988, Nature 333, 208

Wilkes, B.J., Elvis, M.: 1987, Astrophys. J. 323, 243

Wills, B.J.: 1989, in BL Lac Objects, eds. Maraschi, L., Maccacaro, T., Ulrich, M.-H., Springer, Berlin Heidelberg New York, p. 109

Wills, B.J., Netzer, H., Wills, D.: 1985, Astrophys. J. 288, 94

Wilson, A.S.: 1988, Ast. Ap. 206, 41

Wilson, A.S., Heckman, T.M.: 1985, in Astrophysics of Active Galaxies and Quasi-Stellar Objects, ed. Miller, J.S., University Science Books, Mill Valley, p. 39

Wilson, A.S., Meurs, E.J.A.: 1982, Astron. Astrophys. Suppl. 50, 217

Wilson, A.S., Ward, M.J., Haniff, C.A.: 1988, Astrophys. J. 334, 121

Woltjer, L.: 1989, in BL Lac Objects, eds. Maraschi, L., Maccacaro, T., Ulrich, M.-H., Springer, Berlin Heidelberg New York, p. 460 
Worrall, D.M.: 1987, Astrophys. J. 318, 188

Worrall, D.M.: 1989a, in BL Lac Objects, eds. Maraschi, L., Maccacaro, T., Ulrich, M.-H., Springer, Berlin Heidelberg New York, p. 305

Worrall, D.M.: 1989 b, Proc. of the 23rd ESLAB Symposium (ESA SP-296), p. 719

Worrall, D.M. et al.: 1986, Astrophys. J. 303, 589

Wright, G.S., Joseph, R.D., Meikle, W.P.S.: 1984, Nature 309, 430 